\title{
THE RUBIN-STARK CONJECTURE FOR A SPECIAL CLASS OF FUNCTION FIELD EXTENSIONS
}

\author{
Cristian D. Popescu ${ }^{1}$
}

\begin{abstract}
We prove a strong form of the Brumer-Stark Conjecture and, as a consequence, a strong form of Rubin's integral refinement of the abelian Stark Conjecture, for a large class of abelian extensions of an arbitrary characteristic $p$ global field $k$. This class includes all the abelian extensions $K / k$ contained in the compositum $k_{p \infty}:=k_{p} \cdot k_{\infty}$ of the maximal pro- $p$ abelian extension $k_{p} / k$ and the maximal constant field extension $k_{\infty} / k$ of $k$, which happens to sit inside the maximal abelian extension $k^{\text {ab }}$ of $k$ with a quasi-finite index. This way, we extend the results obtained by the present author in [P2].
\end{abstract}

\section{Introduction}

In $[\mathrm{Ru}]$, Rubin formulated an integral refinement of Stark's Conjecture (see [St] and [Ta4]), for abelian Artin $L$-functions of arbitrary order of vanishing at $s=0$, in the case of number fields (i.e. characteristic 0 global fields). In [P2] (see also $[\mathrm{P} 1])$, we extended the Rubin-Stark Conjecture to the case of function fields over finite fields (i.e. characteristic $p$ global fields). In [P2] (see also [P6]) we show that, in the case of function fields, for every prime number $\ell$, the $\ell$-primary component of a strong form of the Rubin-Stark Conjecture is a consequence of the $\ell$-primary component of a strong form of the Brumer-Stark Conjecture, involving Fitting ideals rather than annihilators of ideal class-groups viewed as modules over the appropriate integral group-rings. In [P2], we proved the $\ell$-primary component of the Strong Brumer-Stark Conjecture for all primes $\ell$ not dividing the order of the Galois group $G(K / k)$ of the abelian extension $K / k$ in question, thereby proving the Strong Rubin-Stark Conjecture up to primes dividing the order of the Galois group, for arbitrary abelian extensions of characteristic $p$ function fields. In the case where $K / k$ is a constant field extension, we proved the full Strong Brumer-Stark Conjecture and thereby proved the full Strong Rubin-Stark Conjecture (see [P2]). However, in [P5] (see also [P6]) we show that, in general, the Strong Brumer-Stark Conjecture is false in the function field case. Therefore, it cannot be expected that the proof of the Rubin-Stark Conjecture in full generality can be achieved via a proof of the Strong Brumer-Stark Conjecture.

In this paper, we extend the results of [P2] in several directions. Firstly, we show that, if the prime $\ell$ is different from the characteristic $p$ and the (integral) $\ell$-adic homology groups $\mathrm{H}_{i}\left(\overline{X_{K}}, \mathbb{Z}_{\ell}\right)$ of the geometric smooth projective curve $\overline{X_{K}}$

\footnotetext{
${ }^{1}$ Research on this project was partially supported by NSF grant DMS-0350441.

1991 Mathematics Subject Classification. 11R42, 11R58, 11R27.

Key words and phrases. L-functions, regulators, units, class-groups, class-field theory, $\ell$-adic homology, function fields.
} 
associated to $K$ are $G(K / k)$-cohomologically trivial, then the $\ell$-primary components of the Strong Brumer-Stark and Strong Rubin-Stark Conjectures hold true (see Theorems 3.1 and 3.2). We show that this $G(K / k)$-cohomological triviality property at the level of $\ell$-adic homology is satisfied if and only if the extension $K / k$ is $\ell$-constant (see Proposition 2.3.1 and Definition 2.3.2). For a given $\ell$, the class of $\ell$-constant extensions strictly includes the classes of constant field extensions and extensions whose degree is not divisible by $\ell$, both covered in [P2]. Secondly, we use results obtained by Tan in $[\mathrm{T}]$ on the $p$-primary component of a conjecture of Gross, to write an explicit proof of the $p$-primary component of the Rubin-Stark Conjecture for arbitrary abelian extensions $K / k$ in characteristic $p$ (see Theorem 4.3). This extends our results on the $p$-primary component of the Strong Brumer-Stark and Strong Rubin-Stark Conjectures in the case where $K / k$ is either a constant field extension, or an extension whose degree is not divisible by $p$, obtained in [P2]. Thirdly, since every finite extension $K / k$ contained in the field compositum $k_{p \infty}:=k_{p} \cdot k_{\infty}$ of the maximal abelian pro- $p$ extension $k_{p}$ of $k$ and the maximal constant field extension $k_{\infty}$ of $k$ is $\ell$-constant, for all primes $\ell \neq p$ (see $\S 5$ ), we prove the full Rubin-Stark Conjecture and its strong form at primes different from $p$, for all such extensions (see Theorem 5.2). This result settles the Rubin-Stark Conjecture in characteristic $p$ for a very large class of abelian extensions of a given field $k$, as $k_{p \infty}$ happens to sit inside the maximal abelian extension $k^{\mathrm{a} b}$ of $k$ with a quasi-finite index, according to Proposition 5.3 below. Finally, in $\S 6$ we show that every extension $K / k$, such that $K \subseteq k_{p \infty}$, is admissible (see $\S 2.2$ for the definition) and, consequently, the Rubin-Stark Conjecture and a slightly weaker integral refinement of Stark's Conjecture, formulated by the present author in [P4], are equivalent for $K / k$.

The methods employed in this paper are mostly Galois-cohomological and rely to a great extent on the $\ell$-adic homological interpretation of Artin's $L$-functions for characteristic $p$ global fields, essentially due to André Weil and beautifully rewritten in modern language and in the context of Stark's Conjectures by John Tate with Pierre Deligne's assistance in [Ta4].

Acknowledgement. The results described in this paper were obtained by the present author in the Summer of 1998. An early version of this manuscript was written in 1999. It is a pleasure to thank John Tate, David Hayes, and Jean-Pierre Serre for stimulating feedback on our presentation of these results in the number theory seminars and workshops at U. Texas Austin (1998), U. Mass. Amherst (1999), and the Oberwolfach (2002). Also, we would like to thank the referee for his careful reading of this manuscript as well as his many useful suggestions and corrections.

This introduction would be incomplete without mentioning the important recent work of David Burns and his collaborators (see [Bu1-3], [BuGr]) on the Equivariant Tamagawa Number Conjecture (ETNC) for Tate motives and its links to the RubinStark Conjecture and a Conjecture of Gross. In particular, in [Bu1] Burns shows that, under certain hypotheses of homological nature, the ETNC for Tate motives implies a refined version of the Rubin-Stark and Gross Conjectures, in both the function field and number field case. In [Bu3] (preliminary version, 2004), of whose existence we became aware after the final version of our manuscript was completed, the author shows that, among other things, the $\ell$-primary part of ETNC for Tate motives is true in characteristic $p$ and, as a consequence, proves the $\ell$-primary part of the Rubin-Stark Conjecture in characteristic $p$, for all primes $\ell \neq p$. When 
combined with the results in the present paper, this leads to a proof of the full Rubin-Stark Conjecture in characteristic $p$. Our results were obtained several years earlier and with methods completely different from those used by Burns.

\section{Notations, definitions and conjectures}

1.1. Notations. Throughout this paper, $K / k$ will denote an abelian extension of characteristic $p$ global fields (i.e. finite extensions of $\mathbb{F}_{p}(T)$ ), of Galois group $G:=\operatorname{Gal}(K / k)$. We denote by $\widehat{G}$ the group of complex valued characters of $G$. The group of roots of unity in $K^{\times}$is denoted by $\mu_{K}$. The constant field of $k$ is denoted by $\mathbb{F}_{q}$, where $q:=p^{n}$, for some $n \in \mathbb{N}_{\geq 1}$. For a prime $v$ in $k$, we denote by $\mathbb{F}_{q}(v)$ its residue field and by $d_{v}$ its degree over $\mathbb{F}_{q}$, i.e. $d_{v}:=\left[\mathbb{F}_{q}(v): \mathbb{F}_{q}\right]$. Although, in general, the exact field of constants of $K$ is larger than $\mathbb{F}_{q}$, if $w$ is a prime in $K$, we denote by $d_{w}$ its degree over $\mathbb{F}_{q}$ (i.e. $d_{w}:=\left[\mathbb{F}_{q}(w): \mathbb{F}_{q}\right]$ ), not over the exact field of constants of $K$. For $w$ as above, $\mathbf{N} w:=\left|\mathbb{F}_{q}(w)\right|=q^{d_{w}}$. We denote by $|\cdot|_{w}$ the normalized metric associated to $w$, defined by

$$
|x|_{w}:=(\mathbf{N} w)^{-\operatorname{ord}_{w}(x)},
$$

for all $x \in K^{\times}$. Let $S$ and $T$ be two finite, nonempty sets of primes in $k$. For a finite extension $K^{\prime} / k, S_{K^{\prime}}$ and $T_{K^{\prime}}$ will denote the sets of primes in $K^{\prime}$ dividing primes in $S$ and $T$, respectively. For the moment, we require that the sets $S$ and $T$ satisfy the following set of hypotheses.

\section{Hypotheses $\left(\mathbf{H}_{0}\right)$}

(1) $S$ contains at least all the primes which ramify in $K / k$.

(2) $T \cap S=\emptyset$.

For a finite extension $K^{\prime} / k, O_{K^{\prime}, S}$ will denote its ring of $S_{K^{\prime}}$-integers, $U_{K^{\prime}, S}:=$ $O_{K^{\prime}, S}^{\times}$is the group of $S_{K^{\prime}}$-units in $K^{\prime}$, and $A_{K^{\prime}, S}$ the ideal-class group of $O_{K^{\prime}, S}$. For any such $K^{\prime}$, we also define the $(S, T)$-modified group of units and respectively ideal class-group as follows.

$$
\begin{gathered}
U_{K^{\prime}, S, T}:=\left\{x \in U_{K^{\prime}, S} \mid x \equiv 1 \bmod w, \forall w \in T_{K^{\prime}}\right\} . \\
A_{K^{\prime}, S, T}:=\frac{\left\{\text { fractional ideals of } O_{K^{\prime}, S} \text { coprime to } T_{K^{\prime}}\right\}}{\left\{x \cdot O_{K^{\prime}, S} \mid x \equiv 1 \bmod w, \forall w \in T_{K^{\prime}}\right\}} .
\end{gathered}
$$

The reader will notice right away that, since $T \neq \emptyset$, the groups $U_{K^{\prime}, S, T}$ have no $\mathbb{Z}$-torsion. For simplicity, we will set $U_{S}:=U_{K, S}, A_{S}:=A_{K, S}, U_{S, T}:=U_{K, S, T}$, and $A_{S, T}:=A_{K, S, T}$. Since $S_{K}$ and $T_{K}$ are $G$-invariant, these groups are endowed with natural $\mathbb{Z}[G]$-module structures.

Throughout this paper, if $M$ is a $\mathbb{Z}[G]$-module and $R$ is a commutative ring with 1 , then $R M$ denotes the tensor product $R \otimes_{\mathbb{Z}} M$, endowed with the usual $R[G]$ module structure. Also, $\widetilde{M}$ denotes the image of $M$ via the canonical morphism $M \longrightarrow \mathbb{Q} M$.

1.2. The $G$-equivariant $L$-function. For $K / k, S$, and $T$ as above, and any $\chi$ in $\widehat{G}$, let $L_{S}(\chi, s)$ denote, as usual, the $L$-function associated to $\chi$ with Euler factors at primes in $S$ removed, of the complex variable $s$ (see [Ta4] for the precise 
definition). This is a complex valued function, holomorphic everywhere if $\chi$ is nontrivial, and holomorphic outside $s=1$, with a pole of order 1 at $s=1$ if $\chi$ is the trivial character. With the help of these $L$-functions, one can define

$\Theta_{S, T}: \mathbb{C} \longrightarrow \mathbb{C}[G], \quad \Theta_{S, T}(s):=\prod_{v \in T}\left(1-\sigma_{v}^{-1} \cdot(\mathbf{N} v)^{1-s}\right) \cdot\left(\sum_{\chi \in \widehat{G}} L_{S}(\chi, s) \cdot e_{\chi^{-1}}\right)$,

where $\sigma_{v}$ denotes the Frobenius morphism associated to $v$ in $G$ and $e_{\chi^{-1}}:=$ $1 /|G| \sum_{\sigma \in G} \chi(\sigma) \cdot \sigma$ is the idempotent element associated to $\chi^{-1}$ in $\mathbb{C}[G]$. The function $\Theta_{S, T}$ is the so-called $(S, T)$-modified $G$-equivariant $L$-function. If the group-ring $\mathbb{C}[G]$ is viewed in the obvious manner as a direct product of $|G|$ copies of $\mathbb{C}$, then the projections of $\Theta_{S, T}$ onto the various components of $\mathbb{C}[G]$ with respect to this product decomposition are holomorphic everywhere, as functions of the complex variable $s$. Let us fix an integer $r \geq 0$. We associate to $K / k, S, T$ and $r$ as above the following set of hypotheses, which extend $\left(\mathrm{H}_{0}\right)$ above.

Hypotheses $\left(\mathbf{H}_{r}\right)$

(1) $S$ contains at least all the primes which ramify in $K / k$.

(2) $\operatorname{card}(S) \geq r+1$.

(3) $S$ contains at least $r$ distinct primes which split completely in $K / k$.

(4) $T \cap S=\emptyset$.

If the set of data satisfies the set of hypotheses $\left(\mathrm{H}_{r}\right)$, then the orders of vanishing $\operatorname{ord}_{s=0}$ at $s=0$ of the associated standard and $G$-equivariant $L$-functions satisfy

$$
\operatorname{ord}_{s=0} L_{S}(\chi, s) \geq r, \quad \operatorname{ord}_{s=0} \Theta_{S, T}(s) \geq r,
$$

for all $\chi \in \widehat{G}$ (see [Ta4] for a proof.) Under the hypotheses $\left(\mathrm{H}_{r}\right)$, we let

$$
\Theta_{S, T}^{(r)}(0):=\lim _{s \rightarrow 0} \frac{1}{s^{r}} \Theta_{S, T}(s)
$$

denote the coefficient of $s^{r}$ in the Taylor expansion of $\Theta_{S, T}(s)$ at $s=0$. Also, if $M$ is a $\mathbb{Z}[G]$-module, we let

$$
M_{r, S}:=\left\{m \in \widetilde{M} \mid e_{\chi} \cdot m=0 \text { in } \mathbb{C} M \text {, for all } \chi \in \widehat{G} \text { with } \operatorname{ord}_{s=0} L_{S}(\chi, s)>r\right\} .
$$

1.3. Determinants, characteristic polynomials and Fitting ideals. In this section, we remind the reader of a few basic facts of commutative and homological algebra, needed in the homological interpretation of the $G$-equivariant $L$-function (see next section) and throughout the paper. For proofs and additional details, the reader can consult [Sw] (Part II, Chapter 8), [MW] (Appendix), and $\S 1.2$ of [P2]. Let $R$ be a commutative, Noetherian ring with 1 . If $P$ is a finitely generated, projective $R$-module, then $\operatorname{rk}_{P}: \operatorname{Spec}(R) \longrightarrow \mathbb{Z}_{\geq 0}$ is the locally constant rank function associated to $P$, given by $\operatorname{rk}_{P}(\mathfrak{p})=\operatorname{rank}_{R_{\mathfrak{p}}}\left(P_{\mathfrak{p}}\right)$, for all $\mathfrak{p} \in \operatorname{Spec}(R)$, where $R_{\mathfrak{p}}$ and $P_{\mathfrak{p}}$ are the localizations of $R$ and $P$ at $\mathfrak{p}$, respectively. If $Q$ is a projective $R$-module of rank 1 (i.e. $\operatorname{rk}_{Q}(\mathfrak{p})=1$, for all $\mathfrak{p} \in \operatorname{Spec}(R)$ ), then we let $Q^{-1}:=\operatorname{Hom}_{R}(Q, R)$ denote its inverse (which is a projective $R$-module of rank 1 as well). We have canonical $R$-module isomorphisms

$$
\operatorname{Hom}_{R}(Q, Q) \stackrel{\xi_{Q}}{\longrightarrow} Q^{-1} \otimes_{R} Q \stackrel{\mathrm{eval}_{Q}}{\sim} R,
$$


where $\xi_{Q}^{-1}(f \otimes x)(y)=f(x) y$ and $\operatorname{eval}_{Q}(f \otimes x)=f(x)$, for all $f \in Q^{-1}$, and $x, y \in Q$. As the reader will note right away, the composition $\operatorname{eval}_{Q} \circ \xi_{Q}$ is the unique $R-$ module morphism which takes the identity endomorphism of $Q$ into $1_{R} \in R$.

Let $1_{R}=\sum_{i=1}^{n} e_{i}$ be the decomposition of the identity element in $R$ into a sum of indecomposable orthogonal idempotents $e_{i} \in R$, with $i=1, \ldots, n$. This corresponds to a decomposition of $R$ as a direct sum of rings $R=\bigoplus_{i=1}^{n} R \cdot e_{i}$, and also to a decomposition $\operatorname{Spec}(R)=\cup_{i=1}^{n} \operatorname{Spec}\left(R \cdot e_{i}\right)$ as a disjoint union of Zariski irreducible open and closed subsets $\operatorname{Spec}\left(R \cdot e_{i}\right)$ of $\operatorname{Spec}(R)$. Let $r: \operatorname{Spec}(R) \longrightarrow \mathbb{Z}_{>0}$ be a fixed locally constant function. Then $r$ is constant, say equal to $r_{i} \in \mathbb{Z}_{\geq 0}$, when restricted to $\operatorname{Spec}\left(R \cdot e_{i}\right)$, for all $i=1, \ldots, n$. Therefore, if $P$ is an arbitrary finitely generated projective module, one can define

$$
\bigwedge_{R}^{r} P:=\bigoplus_{i=1}^{n} \bigwedge_{R \cdot e_{i}}^{r_{i}} e_{i} P,
$$

which is also projective and finitely generated over $R$. In particular, this allows us to define unambiguously $\bigwedge_{R}^{\mathrm{rk}_{P}} P$, for any finitely generated, projective $R$-module $P$. This is a well defined rank 1 projective $R$-module, satisfying $\left(\bigwedge_{R}^{\mathrm{rk}_{P}} P\right)_{\mathfrak{p}} \stackrel{\sim}{\longrightarrow}$ $\bigwedge_{R_{\mathfrak{p}}}^{\mathrm{rk}_{P}(\mathfrak{p})} P_{\mathfrak{p}}$, for all $\mathfrak{p} \in \operatorname{Spec}(R)$. In [Sw] (Part II, Chapter 8), the module $\bigwedge_{R}^{\mathrm{rk}_{P}} P$ is called the determinant of $P$ and $\operatorname{denoted} \operatorname{det}_{R}(P)$. If $P$ and $Q$ are finitely generated $R$-modules and $f \in \operatorname{Hom}_{R}(P, Q)$, then, for any locally constant function $r: \operatorname{Spec}(R) \longrightarrow \mathbb{Z}_{\geq 0}$, one defines in the obvious way $\bigwedge^{r} f \in \operatorname{Hom}_{R}\left(\bigwedge_{R}^{r} P, \bigwedge_{R}^{r} Q\right)$.

Definition 1.3.1 (determinants). Let $P$ be a finitely generated, projective $R-$ module, and let $f \in \operatorname{Hom}_{R}(P, P)$. The determinant $\operatorname{det}_{R}(f)$ is defined by

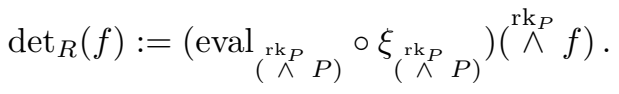

It is very easy to show that, if $R, P$ and $f$ are as above, and $\iota: R \longrightarrow R^{\prime}$ is a morphism of commutative, Noetherian rings with 1 , then we have an equality $\operatorname{det}_{R^{\prime}}\left(f \otimes \mathbf{1}_{R^{\prime}}\right)=\iota\left(\operatorname{det}_{R}(f)\right)$.

Now, let us assume that $R, P$ and $f$ are as above, and let $u$ be a variable (relative to $R$.) Then $P \otimes_{R} R[u]$ is a projective, finitely generated $R[u]$-module.

Definition 1.3.2 (characteristic polynomials). For $R, P, f$ and $u$ as above, the characteristic polynomial of $f$ of variable $u$ is defined to be

$$
\operatorname{char}_{f}(u):=\operatorname{det}_{R[u]}\left(\mathbf{1}_{P} \otimes u-f \otimes \mathbf{1}_{R[u]} \mid P \otimes_{R} R[u]\right),
$$

where $\mathbf{1}_{P} \otimes u$ is the $R[u]$-linear endomorphism of $P \otimes_{R} R[u]$ acting as the identity on $P$ and as multiplication by $u$ on $R[u]$ and $f \otimes \mathbf{1}_{R[u]}$ is defined similarly.

In $\S 1.4$, the following determinant will prove to be useful.

$$
\operatorname{det}_{R}(1-f u \mid P):=\operatorname{det}_{R[u]}\left(\mathbf{1}_{P} \otimes \mathbf{1}_{R[u]}-f \otimes u \mid P \otimes_{R} R[u]\right) .
$$

The determinant above uniquely determines and is determined by $\operatorname{char}_{f}(u)$. We leave the precise relation between the two as an exercise for the interested reader. 
Now, let $M$ be a finitely generated $R$-module. Let us assume that we have an exact sequence of finitely generated $R$-modules

$$
S \stackrel{j}{\rightarrow} P \rightarrow M \rightarrow 0
$$

with $P$ projective. Then, one obtains morphisms of $R$-modules

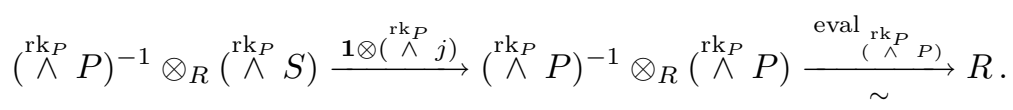

Definition 1.3.3 (Fitting ideals). With notations as above, the (first) Fitting ideal $\mathrm{Fit}_{R}(M)$ of $M$ over $R$ is defined to be the image of the composition of the two morphisms above, i.e.

$$
\operatorname{Fit}_{R}(M):=\operatorname{Im}\left(\operatorname{eval}_{\left(\wedge_{P} P\right)} \circ\left(\mathbf{1} \otimes \stackrel{\mathrm{rk}_{P}}{\wedge} j\right)\right),
$$

and it does not depend on the choice of $S, P$ and $j$.

It is very easy to show that the definition above is equivalent with the more classical definitions of the Fitting ideal of a finitely generated module $M$ presented in the Appendix of [MW] and $\S 1.2$ of [P2], for example. In particular, if $S=P$, it is easy to see that $\operatorname{det}_{R}(j) \in \operatorname{Fit}_{R}(M)$. If $S=P$ and $j$ is injective, i.e. if we have an exact sequence of $R$-modules

$$
0 \rightarrow P \stackrel{j}{\rightarrow} P \rightarrow M \rightarrow 0,
$$

with $P$ projective, then $\operatorname{Fit}_{R}(M)=\operatorname{det}_{R}(M) \cdot R$. We refer the reader to the Appendix of $[\mathrm{MW}]$ and $\S 1.2$ of [P2] for the main properties of the Fitting ideals needed in the present context. Here, we remind the reader of two very important such properties. Namely, if

$$
0 \rightarrow S \rightarrow M \rightarrow N \rightarrow 0
$$

is an exact sequence of finitely generated $R$-modules, then

$$
\operatorname{Fit}_{R}(M) \subseteq \operatorname{Fit}_{R}(N), \quad \operatorname{Fit}_{R}(S) \cdot \operatorname{Fit}_{R}(N) \subseteq \operatorname{Fit}_{R}(M) .
$$

Also, if $\iota: R \longrightarrow R^{\prime}$ is a morphism of Noetherian rings with 1 , and $M$ is a finitely generated $R$-module, then $\operatorname{Fit}_{R^{\prime}}\left(M \otimes_{R} R^{\prime}\right)=\iota\left(\operatorname{Fit}_{R}(M)\right)$.

1.4. The homological interpretation of the $G$-equivariant $L$-function. In this section, we remind the reader the $\ell$-adic homological interpretation of the $(S, T)$-modified $G$-equivariant $L$-functions $\Theta_{S, T}(s)$. The reader can consult [Ta4], [P2], 1.7 , and [P6], 33.1 for the details. Throughout this section, we assume that the set of data $(K / k, S, T)$ satisfies the set of hypotheses $\left(\mathrm{H}_{0}\right)$. Let $X_{K} \stackrel{\pi}{\longrightarrow} X_{k}$ be a $G$-cover of smooth, projective schemes of dimension 1 over $\operatorname{Spec}\left(\mathbb{F}_{q}\right)$, such that the field of rational functions of $X_{K}$ and $X_{k}$ are $K$ and $k$, respectively, and such that $\pi$ induces the inclusion $k \subseteq K$ at the level of fields of rational functions. The schemes $X_{K}$ and $X_{k}$ are unique up to $\operatorname{Spec}\left(\mathbb{F}_{q}\right)$-isomorphism of schemes. Let $\mathbb{F}$ denote an algebraic closure of $\mathbb{F}_{q}$ and let $\overline{X_{K}}$ and $\overline{X_{k}}$ be the smooth, projective 
schemes obtained from $X_{K}$ and $X_{k}$, respectively, by extending scalars from $\mathbb{F}_{q}$ to $\mathbb{F}$, i.e.

$$
\overline{X_{K}}:=X_{K} \times_{\operatorname{Spec}\left(\mathbb{F}_{q}\right)} \operatorname{Spec}(\mathbb{F}), \quad \overline{X_{k}}:=X_{k} \times_{\operatorname{Spec}\left(\mathbb{F}_{q}\right)} \operatorname{Spec}(\mathbb{F}) .
$$

Then, $\overline{X_{K}} \stackrel{\pi \times \mathbf{1}_{\mathbb{P}}}{\longrightarrow} \overline{X_{k}}$ is a $G$-cover of smooth, projective schemes of dimension 1 over $\operatorname{Spec}(\mathbb{F})$. The scheme $\overline{X_{K}}$ is endowed with the natural $G$-action and the action of its $q$-power geometric Frobenius endomorphism $F$. The $G$-action and $F$-action commute and are both $\operatorname{Spec}\left(\mathbb{F}_{q}\right)$-algebraic. Therefore, for every prime number $\ell$, $F$ induces $\mathbb{Z}_{\ell}[G]$-linear endomorphisms of the $\ell$-adic étale homology groups of $\overline{X_{K}}$,

$$
\mathrm{H}_{i}\left(\overline{X_{K}}, \mathbb{Z}_{\ell}\right) \stackrel{F_{*}}{\longrightarrow} \mathrm{H}_{i}\left(\overline{X_{K}}, \mathbb{Z}_{\ell}\right) \text {, for all } i=0,1,2 .
$$

For the definitions and properties of the $\ell$-adic homology groups $\mathrm{H}_{i}\left(\overline{X_{K}}, \mathbb{Z}_{\ell}\right), i=$ $0,1,2$, the reader may consult [P2], $\S 1.7$ or [Ta4]. If $v$ is a prime in $S$, we denote by $I_{v}$ and $G_{v}$ its inertia and decomposition group in $K / k$, respectively. We let

$$
N_{I_{v}}:=\sum_{\sigma \in I_{v}} \sigma .
$$

We denote by $\widetilde{\sigma_{v}}$ a fixed lift in $G_{v}$ of the Frobenius morphism $\sigma_{v} \in G_{v} / I_{v}$ of $v$. Obviously, the element $N_{I_{v}} \cdot{\widetilde{\sigma_{v}}}^{-1} \in \mathbb{Z}[G]$ does not depend on the chosen lift $\widetilde{\sigma_{v}}$ of $\sigma_{v}$. We let

$$
\gamma_{S}(s):=\prod_{v \in S}\left(1-\frac{1}{\left|I_{v}\right|} N_{I_{v}} \cdot{\widetilde{\sigma_{v}}}^{-1} \cdot(\mathbf{N} v)^{-s}\right), \quad \delta_{T}(s):=\prod_{v \in T}\left(1-\sigma_{v}^{-1} \cdot(\mathbf{N} v)^{1-s}\right),
$$

and view them as holomorphic functions of complex variable $s$, taking values in $\mathbb{C}[G]$. The following theorem is proved in $[\mathrm{Ta} 4]$.

Theorem 1.4.1 (Weil-Grothendieck). If the set of data $(K / k, S, T)$ satisfies hypotheses $\left(H_{0}\right)$, then we have an equality of holomorphic functions

$$
\Theta_{S, T}(s)=\delta_{T}(s) \cdot \gamma_{S}(s) \cdot \prod_{i=0}^{2} \operatorname{det}_{\mathbb{Q}_{\ell}[G]}\left(1-q^{-s} \cdot F_{*} \mid \mathrm{H}_{i}\left(\overline{X_{K}}, \mathbb{Z}_{\ell}\right) \otimes_{\mathbb{Z}_{\ell}} \mathbb{Q}_{\ell}\right)^{(-1)^{i+1}},
$$

for all prime numbers $\ell \neq p$.

Remark. The reader will note right away that, since the rings $\mathbb{Q}_{\ell}[G]$ are isomorphic to finite direct sums of fields (finite extensions of $\mathbb{Q}_{\ell}$ ), the $\mathbb{Q}_{\ell}[G]$-modules $\mathrm{H}_{i}\left(\overline{X_{K}}, \mathbb{Z}_{\ell}\right) \otimes_{\mathbb{Z}_{\ell}} \mathbb{Q}_{\ell}$ are projective, for all $i=0,1,2$, and consequently the determinants involved in Theorem 1.4.1 make sense and are defined as in $\S 1.3$ (Definition 1.3.1) above. Moreover, if, for a given prime number $\ell \neq p$, the $\mathbb{Z}_{\ell}[G]$-modules $\mathrm{H}_{i}\left(\overline{X_{K}}, \mathbb{Z}_{\ell}\right)$ happen to be projective, then the extension of scalars property of the determinant (see §1.3) combined with Theorem 1.4.1 lead to an equality

$$
\Theta_{S, T}(s)=\delta_{T}(s) \cdot \gamma_{S}(s) \cdot \prod_{i=0}^{2} \operatorname{det}_{\mathbb{Z}_{\ell}[G]}\left(1-q^{-s} \cdot F_{*} \mid \mathrm{H}_{i}\left(\overline{X_{K}}, \mathbb{Z}_{\ell}\right)\right)^{(-1)^{i+1}}
$$

As usual, we denote by $\operatorname{Pic}^{0}\left(\overline{X_{K}}\right)$ and $\operatorname{Pic}^{0}\left(X_{K}\right)$ the Picard groups of classes of divisors of degree 0 on $\overline{X_{K}}$ and $X_{K}$, respectively. We have an obvious equality 
$\operatorname{Pic}^{0}\left(X_{K}\right)=\operatorname{Pic}^{0}(K)$, where $\operatorname{Pic}^{0}(K)$ is the usual arithmetic Picard group of $K$. If $T$ and $S$ are as above, then $T_{K}$ and $S_{K}$ can be identified, as usual, with two (disjoint) sets of closed points on the scheme $X_{K}$. We denote by $\left(\overline{X_{K}}\right)_{T}$ and $\left(\overline{X_{K}}\right)_{S}$ the (finite) sets consisting of closed points of $\bar{X}_{K}$ sitting above points in $T_{K}$ and $S_{K}$, respectively. As in [P2], 33.1 , we denote by $\operatorname{Pic}^{0}\left(X_{K}\right)_{T}$ and $\operatorname{Pic}^{0}\left(\overline{X_{K}}\right)_{T}$ the Picard groups of classes of divisors of degree 0 on $X_{K}$ and $\overline{X_{K}}$, supported away from $T_{K}$ and $\left(\overline{X_{K}}\right)_{T}$, respectively. More precisely, we have

$$
\begin{aligned}
\operatorname{Pic}^{0}\left(X_{K}\right)_{T} & :=\frac{\operatorname{Div}^{0}\left(X_{K} \backslash T_{K}\right)}{\left\{\operatorname{div}(f) \mid f \in K^{\times}, \quad f \equiv 1 \bmod w, \quad \forall w \in T_{K}\right\}}, \\
\operatorname{Pic}^{0}\left(\overline{X_{K}}\right)_{T} & :=\frac{\operatorname{Div}^{0}\left(\overline{X_{K}} \backslash\left(\overline{X_{K}}\right)_{T}\right)}{\left\{\operatorname{div}(f) \mid f \in\left(K \otimes_{\mathbb{F}_{q}} \mathbb{F}\right)^{\times}, \quad f \equiv 1 \bmod w, \quad \forall w \in\left(\overline{X_{K}}\right)_{T}\right\}} .
\end{aligned}
$$

As in $[\mathrm{P} 2], \S 1.7$, the reader should be aware that, since $\overline{X_{K}}$ is not necessarily connected (which translates into the fact that, in general, $K \otimes_{\mathbb{F}_{q}} \mathbb{F}$ is a direct sum of mutually isomorphic fields), the divisor-degree function on $\bar{X}_{K}$ is in fact a divisor-multidegree function. So, a divisor $D$ on $\overline{X_{K}}$ has degree 0 if and only if it has degree 0 when restricted to each of the Zariski-irreducible component of $\overline{X_{K}}$.

1.5. The Rubin-Stark Conjecture. Throughout this section we assume that the data $(K / k, S, T, r)$ satisfies hypotheses $\left(\mathrm{H}_{r}\right)$, for a fixed $r \in \mathbb{Z}_{\geq 0}$. We fix an $r$-tuple $V:=\left(v_{1}, \ldots, v_{r}\right)$ of $r$ distinct primes in $S$ which split completely in $K / k$, and primes $w_{i}$ in $K$, with $w_{i}$ dividing $v_{i}$, for all $i=1, \ldots, r$. Let $W:=\left(w_{1}, \ldots, w_{r}\right)$.

Definition 1.5.1. The $G$-equivariant regulator map associated to $W$ is the unique $\mathbb{Q}[G]$-linear morphism

$$
R_{W}: \mathbb{Q} \underset{\mathbb{Z}[G]}{\stackrel{r}{\wedge}} U_{S, T} \longrightarrow \mathbb{C}[G]
$$

such that, for all $u_{1}, \ldots, u_{r}$ in $U_{S, T}$, we have

$$
\begin{aligned}
R_{W}\left(u_{1} \wedge \cdots \wedge u_{r}\right) & :=\operatorname{det}\left(-\sum_{g \in G} \log \left|u_{i}^{g^{-1}}\right|_{w_{j}} \cdot g\right)= \\
& =(-1)^{r}(\log q)^{r}\left(\prod_{i=1}^{r} d_{v_{i}}\right) \cdot \operatorname{det}\left(\sum_{g \in G} \operatorname{ord}_{w_{i}}\left(u_{j}^{g^{-1}}\right) \cdot g\right),
\end{aligned}
$$

where the determinants are taken in $\mathbb{C}[G]$ and $\mathbb{Z}[G]$, respectively, and $i, j=1, \ldots, r$.

Remark 1. If extended by $\mathbb{C}$-linearity, and properly restricted, $R_{W}$ induces a $\mathbb{C}[G]$-isomorphism $R_{W}:\left(\mathbb{C}^{r} \mathbb{Z}_{\mathbb{Z}[G]} U_{S, T}\right)_{r, S} \stackrel{\sim}{\longrightarrow} \mathbb{C}[G]_{r, S}$ (see [P2], §1.6).

For any $r$-tuple $\left(\phi_{1}, \ldots, \phi_{r}\right) \in \operatorname{Hom}_{\mathbb{Z}[G]}\left(U_{S, T}, \mathbb{Z}[G]\right)^{r}$, there exists a unique $\mathbb{Q}[G]-$ linear morphism

$$
\phi_{1} \wedge \cdots \wedge \phi_{r}: \mathbb{Q} \underset{\mathbb{Z}[G]}{\wedge} U_{S, T} \longrightarrow \mathbb{Q}[G]
$$

such that, for all $u_{1}, \ldots, u_{r}$ in $U_{S, T}$, we have

$$
\phi_{1} \wedge \cdots \wedge \phi_{r}\left(u_{1} \wedge \cdots \wedge u_{r}\right):=\operatorname{det}\left(\phi_{i}\left(u_{j}\right)\right) .
$$

In the last equality, the determinant is taken with respect to all $i=1, \ldots, r$ and all $j=1, \ldots, r$. Please note that since $U_{S, T}$ has no $\mathbb{Z}$-torsion, $U_{S, T}$ can be naturally viewed as a $\mathbb{Z}[G]$-submodule of $\mathbb{Q} U_{S, T}$. 
Definition 1.5.2 (Rubin). The lattice $\Lambda_{S, T}$ is the $\mathbb{Z}[G]$-submodule of $\mathbb{Q} \underset{\mathbb{Z}[G]}{\stackrel{r}{r} U_{S, T}}$ given by

$$
\Lambda_{S, T}:=\left\{\varepsilon \in\left(\mathbb{Q}^{r} \wedge_{\mathbb{Z}[G]} U_{S, T}\right)_{r, S} \mid \begin{array}{l}
\phi_{1} \wedge \ldots \wedge \phi_{r}(\varepsilon) \in \mathbb{Z}[G] \\
\forall \phi_{1}, \ldots, \phi_{r} \in \operatorname{Hom}_{\mathbb{Z}[G]}\left(U_{S, T}, \mathbb{Z}[G]\right)
\end{array}\right\} .
$$

Now, we are ready to formulate Rubin's integral refinement of Stark's Conjecture in this context. In what follows, we refer to the following statement as the RubinStark Conjecture.

CONJECTURE $B(K / k, S, T, r)$ (Rubin). If the set of data $(K / k, S, T, r)$ satisfies hypotheses $\left(\mathrm{H}_{r}\right)$, then there exists a unique element $\varepsilon_{S, T} \in \Lambda_{S, T}$, such that

$$
R_{W}\left(\varepsilon_{S, T}\right)=\Theta_{S, T}^{(r)}(0)
$$

Remark 2. In view of Remark 1 , the existence and uniqueness of $\varepsilon_{S, T}$ in the $\mathbb{C}$ vector space $\left(\mathbb{C}^{r} \wedge_{\mathbb{Z}[G]} U_{S, T}\right)_{r, S}$, satisfying the regulator condition in the conjecture above is clear. In the case of function fields, it is relatively straight-forward to show that this unique $\varepsilon_{S, T}$ belongs in fact to $\left(\mathbb{Q}^{\wedge} \wedge_{\mathbb{Z}[G]} U_{S, T}\right)_{r, S}$ (see [P2] and [P6]). Therefore, in order to prove $B(K / k, S, T, r)$, it would suffice to show that $\varepsilon_{S, T} \in$ $\mathbb{Z}_{(\ell)} \Lambda_{S, T}$, for all prime numbers $\ell$. In general, if $R$ is a subring of $\mathbb{Q}$ (e.g. $R:=\mathbb{Z}_{(\ell)}$, the localization of $\mathbb{Z}$ at a prime number $\ell, R=\mathbb{Z}[1 /|G|]$, or $R=\mathbb{Q}$ ), we denote by $R B(K / k, S, T, r)$ the statement that the unique $\varepsilon_{S, T} \in\left(\mathbb{C}^{\wedge} \wedge_{\mathbb{Z}[G]} U_{S, T}\right)_{r, S}$, satisfying the regulator condition $R_{W}\left(\varepsilon_{S, T}\right)=\Theta_{S, T}^{(r)}(0)$, belongs in fact to $R \Lambda_{S, T}$. Clearly, we have an equivalence

$$
B(K / k, S, T, r) \Longleftrightarrow\left\{\mathbb{Z}_{(\ell)} B(K / k, S, T, r) \text {, for all primes } \ell\right\} .
$$

In the case $r=1$, the Rubin-Stark Conjecture is equivalent to the classical Brumer-Stark Conjecture (see [P6] for the equivalence). In the function field case, the Brumer-Stark Conjecture was proved independently by Deligne, via an interpretation of $G$-equivariant $L$-functions in terms of $\ell$-adic realizations of certain 1-motives (see [Ta4] and [P6]), and Hayes, via the theory of rank 1, sign-normalized Drinfeld modules (see $[\mathrm{H}]$ ). Therefore, we have the following.

Theorem (Deligne, Hayes). If the set of data $(K / k, S, T, 1)$ satisfies hypotheses $\left(H_{1}\right)$, then conjecture $B(K / k, S, T, 1)$ holds true.

Remark 3. We have an obvious inclusion

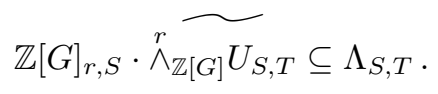

As Rubin shows in $[\mathrm{Ru}]$, the inclusion above is in general strict. However, one can use it to formulate the following strong form of the Rubin-Stark Conjecture. 
CONJECTURE $S B(K / k, S, T, r)$. If the set of data $(K / k, S, T, r)$ satisfies hypotheses $\left(\mathrm{H}_{r}\right)$, then there exists a unique $\varepsilon_{S, T} \in \mathbb{Z}[G]_{r, S} \cdot \stackrel{r}{\wedge}_{\mathbb{Z}[G]} U_{S, T}$, such that

$$
R_{W}\left(\varepsilon_{S, T}\right)=\Theta_{S, T}^{(r)}(0)
$$

As Rubin shows in $[\mathrm{Ru}]$, the conjecture $S B(K / k, S, T, r)$ is in general false in the case of number fields. In [P5] (see also [P6]), we also give a counterexample to this statement in the case of function fields. However, as we will see in this paper, there are many instances in which the strong form of the Brumer-Stark Conjecture holds true in the function field case.

In [P6] it is shown that the following statement implies the Brumer-Stark Conjecture. Consequently, it is called a strong form of the Brumer-Stark Conjecture.

CONJECTURE $S B r S t(K / k, S, T)$. If the set of data $(K / k, S, T)$ satisfies hypotheses $\left(H_{0}\right)$, then

$$
\Theta_{S, T}(0) \in \mathbb{Z}[G]_{0, S} \cdot \operatorname{Fit}_{\mathbb{Z}[G]}\left(A_{S, T}\right),
$$

where Fit $_{\mathbb{Z}[G]}\left(A_{S, T}\right)$ denotes the first Fitting ideal of the $\mathbb{Z}[G]$-module $A_{S, T}$.

Following Remark 2 above, we attach the obvious meaning to the statements $R S B r S t(K / k, S, T)$, for subrings $R$ of $\mathbb{Q}$. In [P5] (see also [P6]), we show that $\mathbb{Z}_{(p)} S B r S t(K / k, S, T)$ is in general false in the case of function fields of characteristic $p$. However, there is a link between the Strong Brumer-Stark and the Strong Rubin-Stark Conjectures in the case of function fields, provided by the following theorem (see [P2] and [P6] for the proof), and which can sometimes be used to prove the latter.

Theorem 1.5.3. Assume that the set of data $(K / k, S, T, r)$ satisfies hypotheses $\left(H_{r}\right)$. Let $v_{1}, v_{2}, \ldots, v_{r} \in S$ be $r$ distinct primes which split completely in $K / k$, and let $S_{0}:=S \backslash\left\{v_{1}, v_{2}, \ldots, v_{r}\right\}$. Then, the following hold true.

(1) For all prime numbers $\ell, \mathbb{Z}_{(\ell)} S B r S t\left(K / k, S_{0}, T\right) \Longrightarrow \mathbb{Z}_{(\ell)} S B(K / k, S, T, r)$.

(2) $\operatorname{SBrSt}\left(K / k, S_{0}, T\right) \Longrightarrow S B(K / k, S, T, r)$.

(3) $\mathbb{Z}[1 /|G|] \operatorname{SBrSt}\left(K / k, S_{0}, T\right) \Longleftrightarrow \mathbb{Z}[1 /|G|] S B(K / k, S, T, r)$.

In [P2] (see Theorems 3.1.1, 3.2.1, 4.2.9, 4.3.1), we exploit these links and prove the following.

Theorem 1.5.4. With notations as in Theorem 1.5.3, we have the following.

(1) $\mathbb{Z}[1 /|G|] S B r S t\left(K / k, S_{0}, T\right)$ and $\mathbb{Z}[1 /|G|] S B(K / k, S, T, r)$ hold true.

(2) If $K / k$ is a constant field extension (i.e. $K=k\left(\mu_{K}\right)$ ), then conjectures $\operatorname{SBrSt}\left(K / k, S_{0}, T\right)$ and $S B(K / k, S, T, r)$ hold true.

The main goal of this paper is to extend the theorem above to a considerably larger class of abelian extensions $K / k$ of an arbitrary characteristic $p$ function field $k$.

\section{2. $\mathbb{Z}[G]-$ modules}

2.1 Basic definitions and properties. In the present section, unless otherwise specified, $G$ denotes a general finite, abelian group, $\ell$ denotes a prime number, $L$ is the $\ell$-Sylow subgroup of $G$, and $G=L \times \Delta$ is the canonical direct product decomposition of $G$ corresponding to its direct factor $L$. 
If $M$ is a $\mathbb{Z}[G]-$ module, and $H$ is a subgroup of $G$, we denote by $\widehat{\mathrm{H}}^{i}(H, M)$ the $i$-th Tate cohomology group of $H$ with coefficients in $M$, for all $i \in \mathbb{Z}$. We refer the reader to $[\mathrm{CF}], \S \mathrm{IV}$, for all the properties of Tate cohomology needed in the present context. The $\mathbb{Z}[G]-$ module $M$ is called $G$-cohomologically trivial if $\widehat{\mathrm{H}}^{i}(H, M)=0$, for all subgroups $H$ of $G$ and all $i \in \mathbb{Z}$. In particular, if $M$ is a $\mathbb{Z}_{\ell}[G]$-module, then $M$ is $G$-cohomologically trivial if and only if $M$ is $L$-cohomologically trivial. An important class of $G$-cohomologically trivial modules is that of $G$-coinduced modules, i.e. modules $M$ of the type

$$
M:=R[G] \otimes_{R} M_{0},
$$

where $R$ is a commutative ring, $M_{0}$ is an $R$-module with trivial $G$-action, and $G$ acts on the tensor product above via its canonical action on the left factor. If $D$ is an arbitrary Dedekind domain (in particular a finite extension of $\mathbb{Z}$ or $\mathbb{Z}_{\ell}$ ) and $M$ is a finitely generated $D[G]$-module, then $M$ is $G$-cohomologically trivial if and only if its projective dimension over $D[G]$ is at most 1, i.e. $\operatorname{pd}_{D[G]}(M) \leq 1$. The $D[G]-$ module $M$ is projective (i.e. $\operatorname{pd}_{D[G]}(M)=0$ ) if and only if $M$ is $G$-cohomologically trivial and it has no $D$-torsion.

We fix an embedding of $\mathbb{C}$ into $\mathbb{C}_{\ell}$ (the completion of the algebraic closure $\overline{\mathbb{Q}_{\ell}}$ of $\mathbb{Q}_{\ell}$ ) and view the elements of $\widehat{G}$ as taking values in $\mathbb{C}_{\ell}$. For any subgroup $H$ of $G$, we define $\widehat{H}\left(\mathbb{Q}_{\ell}\right)$ to be the set of orbits of characters in $\widehat{H}$ with respect to the canonical action of $G\left(\mathbb{C}_{\ell} / \mathbb{Q}_{\ell}\right)$ on $\widehat{H}$. As the notation suggests, the elements in $\widehat{H}\left(\mathbb{Q}_{\ell}\right)$ are in one-to-one correspondence with the characters of irreducible $\mathbb{Q}_{\ell^{-}}$ valued linear representations of $H$. In what follows, if $H$ and $\ell$ are given, we fix once and for all a representative in each character orbit belonging to $\widehat{H}\left(\mathbb{Q}_{\ell}\right)$ and will use the same notation for the chosen representative $\chi \in \widehat{H}$ and its orbit in $\widehat{H}\left(\mathbb{Q}_{\ell}\right)$. This will not generate confusion, as our future constructions are independent (up to isomorphisms in the appropriate categories) of the chosen representative of a given orbit of characters in $\widehat{H}\left(\mathbb{Q}_{\ell}\right)$. Whenever $\ell$ and $G$ are fixed, we let $O_{\chi}:=\mathbb{Z}_{\ell}[\chi]$ (the finite, unramified extension of $\mathbb{Z}_{\ell}$ generated by the values of $\chi$ ), for all $\chi \in \widehat{\Delta}\left(\mathbb{Q}_{\ell}\right)$. We have a ring isomorphism

$$
\mathbb{Z}_{\ell}[G] \stackrel{\sim}{\longrightarrow} \bigoplus_{\chi \in \widehat{\Delta}\left(\mathbb{Q}_{\ell}\right)} O_{\chi}[L]
$$

given by the unique $\mathbb{Z}_{\ell}$-linear map sending $\sigma \in G$ to $(\chi(\sigma))_{\chi \in \widehat{\Delta}\left(\mathbb{Q}_{\ell}\right)}$. The rings $O_{\chi}[L]$ are local, with maximal ideals $(\ell, I(L))$, where $I(L)$ is the augmentation ideal in $O_{\chi}[L]$. In fact, for all $\chi \in \widehat{\Delta}\left(\mathbb{Q}_{\ell}\right)$, we have ring-isomorphisms $O_{\chi}[L] \stackrel{\sim}{\longrightarrow}$ $\mathbb{Z}_{\ell}[G] / \operatorname{ker}(\chi)$, where $\chi: \mathbb{Z}_{\ell}[G] \rightarrow O_{\chi}[L]$ is the unique $\mathbb{Z}_{\ell}$-linear map sending all $\sigma \in G$ to $\chi(\sigma)$. Correspondingly, every $\mathbb{Z}_{\ell}[G]-$ module $M$ can be written as a direct sum

$$
M \stackrel{\sim}{\longrightarrow} \bigoplus_{\chi \in \widehat{\Delta}\left(\mathbb{Q}_{\ell}\right)} M^{\chi},
$$

where $M^{\chi}:=M \otimes_{\mathbb{Z}_{\ell}[G]} O_{\chi}[L] \stackrel{\sim}{\longrightarrow} M / \operatorname{ker}(\chi) \cdot M$. If $m \in M$ and $\chi \in \widehat{\Delta}\left(\mathbb{Q}_{\ell}\right)$, we denote by $m^{\chi}$ the $M^{\chi}$-component of the image of $m$ via the above isomorphism. In particular, a $\mathbb{Z}_{\ell}[G]$-module is projective if and only if $M^{\chi}$ is $O_{\chi}[L]$-projective, for all $\chi \in \widehat{\Delta}\left(\mathbb{Q}_{\ell}\right)$. Since the rings $O_{\chi}[L]$ are local, this happens if and only if $M^{\chi}$ is $O_{\chi}[L]$-free, for all $\chi \in \widehat{\Delta}\left(\mathbb{Q}_{\ell}\right)$. 
2.2. Cohomological triviality of the group of roots of unity. In this section, $K / k$ is a finite abelian extension of characteristic $p$ function fields, and $G=G(K / k)$. We give necessary and sufficient conditions for the $G$-cohomological triviality of $\mu_{K}$ and its $\ell$-Sylow subroups $\mu_{K}^{(\ell)}$, respectively, for all prime numbers $\ell$. We start with the following elementary Lemma.

Lemma 2.2.1. Let $H:=G\left(K / k\left(\mu_{K}\right)\right)$. Then $\mu_{K}$ is $G / H$-cohomologically trivial.

Proof. Let $\mathbb{F}_{q}$ and $\mathbb{F}_{q^{n}}$ be the exact fields of constants of $k$ and $K$, respectively, where $n \in \mathbb{Z}_{\geq 1}$. Obviously, we have an equality of $G / H$-modules $\mu_{K}=\mathbb{F}_{q^{n}}^{\times}$. Via the usual restriction map, we have an isomorphism of Galois groups $G / H \stackrel{\sim}{\longrightarrow}$ $G\left(\mathbb{F}_{q^{n}} / \mathbb{F}_{q}\right)$. In particular, $G / H$ is cyclic. We identify $G / H$ and $G\left(\mathbb{F}_{q^{n}} / \mathbb{F}_{q}\right)$ via the isomorphism above. Let $M$ be a subgroup of $G / H$. Since $M$ is cyclic, we have group isomorphisms

$$
\widehat{\mathrm{H}}^{i}\left(M, \mathbb{F}_{q^{n}}^{\times}\right) \stackrel{\sim}{\longrightarrow} \begin{cases}\widehat{\mathrm{H}}^{0}\left(M, \mathbb{F}_{q^{n}}^{\times}\right), & \text {for } i \text { even } \\ \widehat{\mathrm{H}}^{1}\left(M, \mathbb{F}_{q^{n}}^{\times}\right), & \text {for } i \text { odd } .\end{cases}
$$

The theory of Herbrandt quotients applied to the cyclic group $M$ and the finite $M$-module $\mathbb{F}_{q^{n}}^{\times}$implies that we have an equality

$$
\left|\widehat{\mathrm{H}}^{0}\left(M, \mathbb{F}_{q^{n}}^{\times}\right)\right|=\left|\widehat{\mathrm{H}}^{1}\left(M, \mathbb{F}_{q^{n}}^{\times}\right)\right| .
$$

On the other hand, Hilbert's Theorem 90 applied to the cyclic field extension $\mathbb{F}_{q^{n}} / \mathbb{F}_{q}$ implies that $\widehat{\mathrm{H}}^{1}\left(M, \mathbb{F}_{q^{n}}^{\times}\right)=0$. Consequently, we have $\widehat{\mathrm{H}}^{i}\left(M, \mathbb{F}_{q^{n}}^{\times}\right)=0$, for all $i \in \mathbb{Z}$. This concludes the proof of the Lemma.

Proposition 2.2.2. Let $\ell$ be a prime number. The following hold true.

(1) $\mu_{K}$ is G-cohomologically trivial $\Longleftrightarrow \operatorname{gcd}\left(\left|\mu_{K}\right|,\left[K: k\left(\mu_{K}\right)\right]\right)=1$.

(2) $\mu_{K}^{(\ell)}$ is $G$-cohomologically trivial $\Longleftrightarrow \operatorname{gcd}\left(\left|\mu_{K}^{(\ell)}\right|,\left[K: k\left(\mu_{K}^{(\ell)}\right)\right]\right)=1$.

Proof. (1) Let us assume that $\mu_{K}$ is cohomologically trivial. Let $H:=G\left(K / k\left(\mu_{K}\right)\right)$. If $\operatorname{gcd}\left(\left|\mu_{K}\right|,|H|\right)=m>1$, then, since $H$ acts trivially on $\mu_{K}$, we have group isomorphisms

$$
\widehat{\mathrm{H}}^{0}\left(H, \mu_{K}\right) \stackrel{\sim}{\longrightarrow} \mu_{K} / \mu_{K}^{m} \stackrel{\sim}{\longrightarrow} \mathbb{Z} / m \mathbb{Z},
$$

which shows that $\widehat{\mathrm{H}}^{0}\left(H, \mu_{K}\right) \neq 0$, contradicting the $G$-cohomological triviality of $\mu_{K}$. Therefore $\operatorname{gcd}\left(\left|\mu_{K}\right|,|H|\right)=1$.

Now, let us assume that $\operatorname{gcd}\left(\left|\mu_{K}\right|,|H|\right)=1$. This implies right away that $\mu_{K}$ is $H$-cohomologically trivial. Let $M$ be a subgroup of $G$. Therefore, we have $\widehat{\mathrm{H}}^{i}\left(M \cap H, \mu_{K}\right)=0$, for all $i \in \mathbb{Z}$. The inflation-restriction sequence applied to the group $M$, its subgroup $M \cap H$ and the $M$-module $\mu_{K}$, gives exact sequences of groups

$$
0 \rightarrow \widehat{\mathrm{H}}^{i}\left(M / M \cap H, \mu_{K}^{M \cap H}\right) \stackrel{\operatorname{Inf}}{\longrightarrow} \widehat{\mathrm{H}}^{i}\left(M, \mu_{K}\right) \stackrel{\text { Res }}{\longrightarrow} \widehat{\mathrm{H}}^{i}\left(H, \mu_{K}\right),
$$

for all $i \in \mathbb{Z}$. However, since $M / M \cap H$ is a subgroup of $G / H$, and $\mu_{K}^{M \cap H}=\mu_{K}$, Lemma 2.2.1 implies that the left-most cohomology group in the exact sequence above is trivial. Since $\mu_{K}$ is $H$-cohomologically trivial, the right-most cohomology 
group in the exact sequence above is also trivial. This implies that $\widehat{\mathrm{H}}^{i}\left(M, \mu_{K}\right)=0$, for all $i \in \mathbb{Z}$, concluding the proof of (1).

(2) The proof of (2) is almost identical to that of (1). We leave it as an exercise for the interested reader.

Following [P4], we give the following definition.

Definition 2.2.3. Let $\ell$ be a prime number. An abelian extension $K / k$ of function fields of Galois group $G$ is called $\ell$-admissible if $\mu_{K}^{(\ell)}$ is $G$-cohomologically trivial. The extension $K / k$ is called admissible if $\mu_{K}$ is $G$-cohomologically trivial.

Remark. Obviously, $K / k$ is admissible if and only if it is $\ell$-admissible for all prime numbers $\ell$.

2.3 Cohomological triviality of the $\ell$-adic homology groups. We work under the assumptions and notations of the previous section. In this section we give a necessary and sufficient condition for the $G$-cohomological triviality of the $\ell$-adic étale homology groups $\mathrm{H}_{i}\left(\overline{X_{K}}, \mathbb{Z}_{\ell}\right)$, for all $i=0,1,2$.

Proposition 2.3.1. The following are equivalent.

(1) $\mathrm{H}_{i}\left(\overline{X_{K}}, \mathbb{Z}_{\ell}\right)$ is $G$-cohomologically trivial (equivalently, a projective $\mathbb{Z}_{\ell}[G]$ module), for all $i=0,1,2$.

(2) $\ell \nmid\left[K: k\left(\mu_{K}\right)\right]$.

(3) $K / K^{L}$ is a constant field extension, i.e. $K=K^{L}\left(\mu_{K}\right)$.

Proof. First of all, since $\mathrm{H}_{i}\left(\overline{X_{K}}, \mathbb{Z}_{\ell}\right)$ is free as a $\mathbb{Z}_{\ell}$-module, its $G$-cohomological triviality is equivalent to its projectivity as a $\mathbb{Z}_{\ell}[G]$-module.

(1) $\Longrightarrow(2)$. Let $K^{\prime}:=k\left(\mu_{K}\right)$ and let $H=G\left(K / K^{\prime}\right)$. Since the constant fields of $K$ and $K^{\prime}$ are the same, the explicit description of $\ell$-adic homology following Definition 1.7.3.1 in [P2] give an isomorphism of $\mathbb{Z}_{\ell}[H]$-modules

$$
\mathrm{H}_{0}\left(\overline{X_{K}}, \mathbb{Z}_{\ell}\right) \stackrel{\sim}{\longrightarrow} \mathrm{H}_{0}\left(\overline{X_{K^{\prime}}}, \mathbb{Z}_{\ell}\right) .
$$

This shows that $H$ acts trivially on $\mathrm{H}_{0}\left(\overline{X_{K}}, \mathbb{Z}_{\ell}\right)$. Consequently, if $\ell|| H \mid$, then

$$
\widehat{\mathrm{H}}^{0}\left(H, \mathrm{H}_{0}\left(\overline{X_{K}}, \mathbb{Z}_{\ell}\right)\right) \stackrel{\sim}{\longrightarrow} \frac{\mathrm{H}_{0}\left(\overline{X_{K}}, \mathbb{Z}_{\ell}\right)}{|H| \cdot \mathrm{H}_{0}\left(\overline{X_{K}}, \mathbb{Z}_{\ell}\right)} \neq 0 .
$$

This contradicts (1), therefore $\ell \nmid|H|$.

(2) $\Longrightarrow(\mathbf{3})$. Since $\ell \nmid|H|$, the intersection $H \cap L$ is trivial. Galois theory implies that the compositum $K^{L} \cdot K^{\prime}=K^{L} \cdot K^{H}$ equals $K$. Since $K^{\prime}=k\left(\mu_{K}\right)$, this shows that $K=K^{L}\left(\mu_{K}\right)$, which concludes the proof of (3).

(3) $\Longrightarrow(\mathbf{1})$. Since $K / K^{L}$ is a constant field extension, Lemma 4.1.1. in [P2] gives isomorphisms of $\mathbb{Z}_{\ell}[L]$-modules,

$$
\mathrm{H}_{i}\left(\overline{X_{K}}, \mathbb{Z}_{\ell}\right) \stackrel{\sim}{\longrightarrow} \mathrm{H}_{i}\left(\overline{X_{K^{L}}}, \mathbb{Z}_{\ell}\right) \otimes_{\mathbb{Z}_{\ell}} \mathbb{Z}_{\ell}[L],
$$

for all $i=0,1,2$, with $L$ acting trivially on $\mathrm{H}_{i}\left(\overline{X_{K^{L}}}, \mathbb{Z}_{\ell}\right)$ and canonically on $\mathbb{Z}_{\ell}[L]$. The above isomorphisms show that the $L$-modules are $L$-coinduced (see [CF] for the definition) and therefore $L$-cohomologically trivial (see $[\mathrm{CF}]$ ). According to $\S 2.1$, this implies that they are $G$-cohomologically trivial. This concludes the proof of (1) and Proposition 2.3.1. 
Definition 2.3.2. Let $\ell$ be a prime number. A finite abelian extension of function fields $K / k$ is called $\ell$-constant if $K / K^{L}$ is a constant field extension, where $L$ is the $\ell-S y l o w$ subgroup of $G(K / k)$.

Remark. Proposition 2.3.1 above shows that, given a prime number $\ell$, the $\ell$-adic homology groups $\mathrm{H}_{i}\left(\overline{X_{K}}, \mathbb{Z}_{\ell}\right)$ are $G$-cohomologically trivial for all $i=0,1,2$ if and only if the extension $K / k$ is $\ell$-constant. Obviously, $K / k$ is $\ell$-constant at all primes $\ell \nmid|G(K / k)|$. Also, it is immediate that $K / k$ is a constant field extension if and only if it is $\ell$-constant for all prime numbers $\ell$.

We conclude this section with a lemma connecting the classes of $\ell$-constant and $\ell$-admissible extensions.

Lemma 2.3.3. Let $K / k$ be an abelian extension of characteristic $p$ global fields and let $\ell$ be a prime number. The following hold true.

(1) If $K / k$ is $\ell$-constant then $K / k$ is $\ell$-admissible.

(2) If $K / k$ is $\ell$-admissible and $\mu_{K}^{(\ell)} \neq\{1\}$, then $K / k$ is $\ell$-constant.

(3) For a fixed $k$, if $\mu_{k}^{(\ell)}=\{1\}$, there always exists an $\ell$-admissible extension $K / k$ which is not $\ell$-constant.

Proof. (1) Let us assume that $K / k$ is $\ell$-constant. As usual, let $L$ be the $\ell$-Sylow subgroup of $G(K / k)$. Since $K=K^{L}\left(\mu_{K}\right)$, Lemma 2.2.1 implies that $\mu_{K}$ is $L$ cohomologically trivial. Therefore $\mu_{K}^{(\ell)}$ is $L$-cohomologically trivial. Consequently, $\mu_{K}^{(\ell)}$ is $G(K / k)$-cohomologically trivial. Hence $K / k$ is $\ell$-admissible.

(2) Under the assumptions of (2), Proposition 2.2.2(2) implies that $\ell \nmid[K$ : $\left.k\left(\mu_{K}^{(\ell)}\right)\right]$. Consequently $\ell \nmid\left[K: k\left(\mu_{K}\right)\right]$. Proposition 2.3.1(2) implies that $K / k$ is $\ell$-constant.

(3) Let $v_{\infty}$ and $v$ be two distinct primes in $k$, such that $\operatorname{gcd}\left(\ell \cdot(\ell-1), d_{v_{\infty}}\right)=1$ and $\ell \nmid\left[\mathbb{F}_{q}(v): \mathbb{F}_{q}^{\times}\right]$. Class-field theory implies that the maximal abelian extension $k(v)$ of $k$, which is completely split at $v_{\infty}$ and of conductor at most $v$ satisfies

$$
|G(k(v) / k)|=\left|\operatorname{Pic}^{0}(k)\right| \cdot d_{v_{\infty}} \cdot\left|\mathbb{F}_{q}(v)^{\times} / \mathbb{F}_{q}^{\times}\right| .
$$

Therefore, $\ell|| G(k(v) / k) \mid$. We claim that, under the hypothesis $\mu_{k}^{(\ell)}=\{1\}$ the extension $k(v) / k$ is $\ell$-admissible but not $\ell$-constant. This follows immediately from the observation that, since $v_{\infty}$ splits completely in $k(v) / k$, then the maximal constant field contained in $k(v)$ is $k \cdot \mathbb{F}_{q^{d v_{\infty}}}$. Therefore, $\mu_{k(v)}=\mathbb{F}_{q^{d_{\infty}}}^{\times}$. Since $\ell \nmid(q-1)$ and $\operatorname{gcd}\left(\ell \cdot(\ell-1), d_{v_{\infty}}\right)=1$, we have $\mu_{k(v)}^{(\ell)}=\{1\}$. Consequently, $k(v) / k$ is $\ell$-admissible. However, $k(v) / k$ is not $\ell$-constant, since $\ell|| G(k(v) / k) \mid$, but the maximal constant field extension of $k$ contained in $k(v)$ has degree $d_{v_{\infty}}$, which is not divisible by $\ell$.

\section{The $\ell$-primary component of the strong Brumer-Stark and strong Rubin-Stark conjectures in $\ell$-constant extensions}

Throughout this section, $\left(K / k, S_{0}, T\right)$ is a set of data satisfying hypotheses $\left(H_{0}\right)$, $G:=G(K / k), \ell$ is a prime number different from $p:=\operatorname{char}(k), L$ is the $\ell$-Sylow subgroup of $G, G=L \times \Delta$, and $H:=G\left(K / K^{\prime}\right)$, where $K^{\prime}:=k\left(\mu_{K}\right)$. In this section, our main goal is to prove the following theorem and its immediate corollary. 
Theorem 3.1. . If $\ell \nmid\left[K: k\left(\mu_{k}\right)\right]$, then the $\ell$-primary component of the Strong Brumer-Stark Conjecture $\mathbb{Z}_{\ell} \operatorname{SBrSt}\left(K / k, S_{0}, T\right)$ is true, i.e.

$$
\Theta_{S_{0}, T}(0) \in \mathbb{Z}_{\ell}[G]_{0, S_{0}} \cdot \operatorname{Fit}_{\mathbb{Z}_{\ell}[G]}\left(A_{S_{0}, T} \otimes \mathbb{Z}_{\ell}\right) .
$$

The following is an immediate consequence of the above theorem.

Theorem 3.2. Let $(K / k, S, T, r)$ be a set of data satisfying hypotheses $(H)_{r}$. Assume that $\ell$ is a prime different from $p=\operatorname{char}(k)$, such that $\ell \nmid\left[K: k\left(\mu_{K}\right)\right]$. Then, the $\ell$-primary component of the strong Rubin-Stark Conjecture $\mathbb{Z}_{(\ell)} \operatorname{SB}(K / k, S, T, r)$ holds true.

Proof. Let $\left\{v_{1}, v_{2}, \ldots, v_{r}\right\}$ be a set of $r$ distinct primes in $S$ which split completely in $K / k$. Let $S_{0}:=S \backslash\left\{v_{1}, v_{2}, \ldots, v_{r}\right\}$. Then, the set of data $\left(K / k, S_{0}, T\right)$ satisfies hypotheses $\left(H_{0}\right)$. Theorem 3.1 above implies that $\mathbb{Z}_{\ell} \operatorname{SBrSt}\left(K / k, S_{0}, T\right)$ holds true. Consequently, Theorem 1.5.4(1) implies that $\mathbb{Z}_{\ell} \mathrm{SB}(K / k, S, T, r)$ holds true.

Proof of Theorem 3.1. According to $§ 2.1$, we need to show that we have

$$
\Theta_{S_{0}, T}(0)^{\chi} \in\left(\mathbb{Z}_{\ell}[G]_{0, S_{0}}\right)^{\chi} \cdot\left(\operatorname{Fit}_{\mathbb{Z}_{\ell}[G]}\left(A_{S_{0}, T} \otimes \mathbb{Z}_{\ell}\right)\right)^{\chi},
$$

for all $\chi \in \widehat{\Delta}\left(\mathbb{Q}_{\ell}\right)$. Relation (1) above is viewed inside $O_{\chi}[L]$, for all $\chi \in \widehat{\Delta}\left(\mathbb{Q}_{\ell}\right)$. Let us fix $\chi \in \widehat{\Delta}\left(\mathbb{Q}_{\ell}\right)$. Since $\ell \nmid|H|$, we have an inclusion $H \subseteq \Delta$. We divide the proof into two distinct cases.

Case I. Assume that $\chi$ restricted to $H$ is the trivial character $\mathbf{1}_{H}$, i.e.

$$
\chi \in \widehat{\Delta / H}\left(\mathbb{Q}_{\ell}\right) \subseteq \widehat{\Delta}\left(\mathbb{Q}_{\ell}\right) .
$$

Lemma 3.3. Under the assumption above, the following equalities hold inside the group-ring $O_{\chi}[L]$.

(1) $\left(\mathbb{Z}_{\ell}[G]_{0, S_{0}}\right)^{\chi}=\left(\mathbb{Z}_{\ell}[G / H]_{0, S_{0}}\right)^{\chi}$.

(2) $\Theta_{S_{0}, T}(0)^{\chi}=\Theta_{K^{\prime} / k, S_{0}, T}(0)^{\chi}$.

(3) $\left(\operatorname{Fit}_{\mathbb{Z}_{\ell}[G]}\left(A_{S_{0}, T} \otimes \mathbb{Z}_{\ell}\right)\right)^{\chi}=\left(\operatorname{Fit}_{\mathbb{Z}_{\ell}[G / H]}\left(A_{K^{\prime}, S_{0}, T} \otimes \mathbb{Z}_{\ell}\right)\right)^{\chi}$.

Proof of Lemma 3.3. (1) By definition, we have equalities

$$
\left(\mathbb{Z}_{\ell}[G]_{0, S_{0}}\right)^{\chi}=\left(\mathbb{Z}_{\ell}[G]^{\chi}\right)_{0, S_{0}}, \quad\left(\mathbb{Z}_{\ell}[G / H]_{0, S_{0}}\right)^{\chi}=\left(\mathbb{Z}_{\ell}[G / H]^{\chi}\right)_{0, S_{0}} .
$$

On the other hand, the canonical projection $\pi: \mathbb{Z}_{\ell}[G] \rightarrow \mathbb{Z}_{\ell}[G / H]$, induced by the restriction map at the level of Galois groups, induces ring isomorphisms

$$
\pi^{\chi}: \mathbb{Z}_{\ell}[G]^{\chi} \stackrel{\sim}{\longrightarrow} \mathbb{Z}_{\ell}[G / H]^{\chi}, \quad \text { for all } \chi \in \widehat{\Delta / H}\left(\mathbb{Q}_{\ell}\right) .
$$

These isomorphisms and the two equalities above, lead to a proof of (1).

(2) It is very easy to show that the inflation property of the Artin $L$-functions implies that the canonical projection $\pi: \mathbb{Z}_{\ell}[G] \rightarrow \mathbb{Z}_{\ell}[G / H]$ sends $\Theta_{S_{0}, T}(0)$ to $\Theta_{K^{\prime} / k, S_{0}, T}(0)$. Consequently, for every $\chi \in \widehat{\Delta / H}\left(\mathbb{Q}_{\ell}\right)$, we have equalities

$$
\Theta_{S_{0}, T}(0)^{\chi}=\left(\pi\left(\Theta_{S_{0}, T}(0)\right)\right)^{\chi}=\Theta_{K^{\prime} / k, S_{0}, T}(0)^{\chi},
$$


which concludes the proof of (2).

(3) Since $\ell \nmid H$, the usual norm map $N_{K / K^{\prime}}$ at the level of ideal classes in the extension $K / K^{\prime}$ induces an isomorphism of $\mathbb{Z}_{\ell}[G]$-modules

$$
N_{K / K^{\prime}}:\left(A_{S_{0}, T} \otimes \mathbb{Z}_{\ell}\right)^{H} \stackrel{\sim}{\longrightarrow} A_{K^{\prime}, S_{0}, T} \otimes \mathbb{Z}_{\ell} .
$$

(For example, see Lemma 1.7 in [P3] for the isomorphism above.) Obviously, the isomorphism above induces isomorphisms of $O_{\chi}[L]$-modules

$$
N_{K / K^{\prime}}^{\chi}:\left(A_{S_{0}, T} \otimes \mathbb{Z}_{\ell}\right)^{\chi} \stackrel{\sim}{\longrightarrow}\left(A_{K^{\prime}, S_{0}, T} \otimes \mathbb{Z}_{\ell}\right)^{\chi},
$$

for all $\chi \in \widehat{\Delta / H}\left(\mathbb{Q}_{\ell}\right)$. These isomorphisms induce equalities of Fitting ideals

$$
\text { Fit }_{O_{\chi}[L]}\left(\left(A_{S_{0}, T} \otimes \mathbb{Z}_{\ell}\right)^{\chi}\right)=\text { Fit }_{O_{\chi}[L]}\left(\left(A_{K^{\prime}, S_{0}, T} \otimes \mathbb{Z}_{\ell}\right)^{\chi}\right),
$$

for all $\chi \in \widehat{\Delta / H}\left(\mathbb{Q}_{\ell}\right)$. The extension of scalars property of the Fitting ideals (see \$1.3) applied to the surjective ring morphisms $\mathbb{Z}_{\ell}[G] \rightarrow O_{\chi}[L]$ and $\mathbb{Z}_{\ell}[G / H] \rightarrow$ $O_{\chi}[L]$, leads to the equalities

$$
\begin{array}{r}
\left(\mathrm{Fit}_{\mathbb{Z}_{\ell}[G]}\left(A_{S_{0}, T} \otimes \mathbb{Z}_{\ell}\right)\right)^{\chi}=\operatorname{Fit}_{O_{\chi}[L]}\left(\left(A_{S_{0}, T} \otimes \mathbb{Z}_{\ell}\right)^{\chi}\right), \\
\left(\operatorname{Fit}_{\mathbb{Z}_{\ell}[G / H]}\left(A_{K^{\prime}, S_{0}, T} \otimes \mathbb{Z}_{\ell}\right)\right)^{\chi}=\operatorname{Fit}_{O_{\chi}[L]}\left(\left(A_{K^{\prime}, S_{0}, T} \otimes \mathbb{Z}_{\ell}\right)^{\chi}\right),
\end{array}
$$

for all $\chi \in \widehat{\Delta / H}\left(\mathbb{Q}_{\ell}\right)$. Equality (3) follows by combining the last two sets of equalities.

We are now ready to conclude the proof of Theorem 3.1 in Case I. Theorem 1.5.4(2) applied to the constant field extension $K^{\prime} / k$ gives

$$
\Theta_{K^{\prime} / k, S_{0}, T}(0)^{\chi} \in\left(\mathbb{Z}_{\ell}[G / H]_{0, S_{0}}\right)^{\chi} \cdot\left(\operatorname{Fit}_{\mathbb{Z}_{\ell}[G / H]}\left(A_{K^{\prime} / k, S_{0}, T} \otimes \mathbb{Z}_{\ell}\right)\right)^{\chi},
$$

for all $\chi \in \widehat{\Delta / H}\left(\mathbb{Q}_{\ell}\right)$. Consequently, Lemma 3.3 implies that equalities (1) hold true for all $\chi \in \widehat{\Delta / H}\left(\mathbb{Q}_{\ell}\right)$.

Case II. Assume that $\chi$ restricted to $H$ is not the trivial character $\mathbf{1}_{H}$, i.e.

$$
\chi \in \widehat{\Delta}\left(\mathbb{Q}_{\ell}\right) \backslash \widehat{\Delta / H}\left(\mathbb{Q}_{\ell}\right) .
$$

Since, under our present hypotheses, the homology groups $\mathrm{H}_{i}\left(\overline{X_{K}}, \mathbb{Z}_{\ell}\right)$ are projective $\mathbb{Z}_{\ell}[G]$-modules (see Proposition 2.3.1), the remark at the end of $\S 1.4$ implies that we have the following equality in $\mathbb{Q}_{\ell}[G]$

$$
\Theta_{S_{0}, T}(0)=\gamma_{S_{0}}(0) \cdot \delta_{T}(0) \cdot \prod_{i=0}^{2} \operatorname{det}_{\mathbb{Z}_{\ell}[G]}\left(1-F_{*} \mid \mathrm{H}_{i}\left(\overline{X_{K}}, \mathbb{Z}_{\ell}\right)\right)^{(-1)^{i+1}} .
$$

Since $\mathrm{H}_{i}\left(\overline{X_{K}}, \mathbb{Z}_{\ell}\right)^{\chi}$ are projective $O_{\chi}[L]$-modules, for all $\chi \in \widehat{\Delta}\left(\mathbb{Q}_{\ell}\right)$, we also have the following equalities in $O_{\chi}[L]$.

$$
\Theta_{S_{0}, T}(0)^{\chi}=\gamma_{S_{0}}(0)^{\chi} \cdot \delta_{T}(0)^{\chi} \cdot \prod_{i=0}^{2} \operatorname{det}_{O_{\chi}[L]}\left(1-F_{*} \mid \mathrm{H}_{i}\left(\overline{X_{K}}, \mathbb{Z}_{\ell}\right)^{\chi}\right)^{(-1)^{i+1}}
$$


Since $K^{\prime} / k$ is a constant field extension and $K / K^{\prime}$ is a purely geometric extension (i.e. $\mu_{K}=\mu_{K^{\prime}}$ ), Lemma 4.1.1 in [P2] provides us with $\mathbb{Z}_{\ell}[G]$-module isomorphisms

$$
\mathrm{H}_{0}\left(\overline{X_{K}}, \mathbb{Z}_{\ell}\right) \stackrel{\sim}{\longrightarrow} \mathbb{Z}_{\ell}[G / H], \quad \mathrm{H}_{2}\left(\overline{X_{K}}, \mathbb{Z}_{\ell}\right) \stackrel{\sim}{\longrightarrow} \mathbb{Z}_{\ell}[G / H] .
$$

Also, according to loc. cit., via these isomorphisms, the actions of $F_{*}$ on $\mathrm{H}_{0}\left(\overline{X_{K}}, \mathbb{Z}_{\ell}\right)$ and $\mathrm{H}_{2}\left(\overline{X_{K}}, \mathbb{Z}_{\ell}\right)$ are taken into multiplication by $\sigma_{q}^{-1}$ and $q \cdot \sigma_{q}^{-1}$, respectively, where $\sigma_{q}$ is the distinguished generator of $G / H=G\left(K^{\prime} / k\right)$, which satisfies $\sigma_{q}(\zeta)=\zeta^{q}$, for all $\zeta \in \mu_{K}$. ( $\sigma_{q}$ is the so-called $q$-power arithmetic Frobenius morphism.) The isomorphisms above imply that, for all $i=0,2$, and all $\chi \in \widehat{\Delta}\left(\mathbb{Q}_{\ell}\right) \backslash \widehat{\Delta / H}\left(\mathbb{Q}_{\ell}\right)$, we have

$$
\mathrm{H}_{i}\left(\overline{X_{K}}, \mathbb{Z}_{\ell}\right)^{\chi}=0 .
$$

Consequently, for all $i=0,2$, and all $\chi \in \widehat{\Delta}\left(\mathbb{Q}_{\ell}\right) \backslash \widehat{\Delta / H}\left(\mathbb{Q}_{\ell}\right)$, we have

$$
\operatorname{det}_{O_{\chi}[L]}\left(1-F_{*} \mid \mathrm{H}_{i}\left(\overline{X_{K}}, \mathbb{Z}_{\ell}\right)^{\chi}\right)=1 .
$$

Proposition 3.4. Under the above hypotheses, we have

(1) $\gamma_{S_{0}}(0)^{\chi} \in\left(\mathbb{Z}_{\ell}[G]_{0, S_{0}}\right)^{\chi}$.

(2) $\delta_{T}(0)^{\chi} \cdot \operatorname{det}_{O_{\chi}[L]}\left(1-F_{*} \mid \mathrm{H}_{1}\left(\overline{X_{K}}, \mathbb{Z}_{\ell}\right)^{\chi}\right) \in\left(\operatorname{Fit}_{\mathbb{Z}_{\ell}[G]}\left(A_{S_{0}, T} \otimes \mathbb{Z}_{\ell}\right)\right)^{\chi}$.

Proof of Proposition 3.4. (1) Since $K^{\prime} / k$ is a constant field extension, $K^{\prime} / k$ is unramified everywhere. Consequently, the inertia groups $I_{v}$ of all the primes $v \in S_{0}$ are in fact included in $H=G\left(K / K^{\prime}\right)$. Therefore, $\ell \nmid\left|I_{v}\right|$, for all $v \in S_{0}$. Therefore, $\gamma_{S_{0}}(0) \in \mathbb{Z}_{\ell}[G]$. Moreover, we claim that in fact we have

$$
\gamma_{S_{0}}(0) \in \mathbb{Z}_{\ell}[G]_{0, S_{0}} .
$$

Indeed, if $\psi \in \widehat{G}$, such that $\operatorname{ord}_{s=0} L_{S_{0}}(\psi, 0)>0$, then there exists $v_{0} \in S_{0}$ with $\left.\psi\right|_{G_{v_{0}}}=\mathbf{1}_{G_{v_{0}}}$ (see the proof of Lemma 1.2.3 in [P6]). Consequently, we have $\psi\left(1 /\left|I_{v_{0}}\right| \cdot N_{I_{v_{0}}} \cdot{\widetilde{\sigma_{v_{0}}}}^{-1}\right)=1$. Hence, $\psi\left(\gamma_{S_{0}}(0)\right)=0$. This concludes the proof of (1).

(2) According to $\S 3.1$ in $[\mathrm{P} 2]$, we have an exact sequence of $\mathbb{Z}_{\ell}[G]$-modules

$$
0 \rightarrow \mathrm{H}_{1}\left(\overline{X_{K}}, \mathbb{Z}_{\ell}\right) \stackrel{1-F_{*}}{\longrightarrow} \mathrm{H}_{1}\left(\overline{X_{K}}, \mathbb{Z}_{\ell}\right) \rightarrow \operatorname{Pic}^{0}\left(X_{K}\right) \otimes \mathbb{Z}_{\ell} \rightarrow 0,
$$

which gives rise to exact sequences of $O_{\chi}[L]$-modules

$$
0 \rightarrow \mathrm{H}_{1}\left(\overline{X_{K}}, \mathbb{Z}_{\ell}\right)^{\chi} \stackrel{1-F_{*}}{\longrightarrow} \mathrm{H}_{1}\left(\overline{X_{K}}, \mathbb{Z}_{\ell}\right)^{\chi} \rightarrow\left(\operatorname{Pic}^{0}\left(X_{K}\right) \otimes \mathbb{Z}_{\ell}\right)^{\chi} \rightarrow 0,
$$

for all $\chi \in \widehat{\Delta}\left(\mathbb{Q}_{\ell}\right)$. Since $\mathrm{H}_{1}\left(\overline{X_{K}}, \mathbb{Z}_{\ell}\right)$ is a $\mathbb{Z}_{\ell}[G]$-projective module (see Proposition 2.3.1) and consequently $\mathrm{H}_{1}\left(\overline{X_{K}}, \mathbb{Z}_{\ell}\right)^{\chi}$ is an $O_{\chi}[L]$-projective module, we have

$$
\operatorname{det}_{O_{\chi}[L]}\left(1-F_{*} \mid \mathrm{H}_{1}\left(\overline{X_{K}}, \mathbb{Z}_{\ell}\right)^{\chi}\right) \in \operatorname{Fit}_{O_{\chi}[L]}\left(\left(\operatorname{Pic}^{0}\left(X_{K}\right) \otimes \mathbb{Z}_{\ell}\right)^{\chi}\right),
$$

for all $\chi \in \widehat{\Delta}\left(\mathbb{Q}_{\ell}\right)$ (see properties of Fitting ideals in $\S 1.3$ above).

As in [P4], $\S 5.3$, let $\Delta_{T}:=\oplus_{w \in T_{K}} \mathbb{F}_{q}(w)^{\times}$. This finite group comes endowed with a canonical $G$-action (see [P4], $\S 5.3$ or [P2], $\S 3.1$ ) and one has a $\mathbb{Z}[G]$-module isomorphism

$$
\Delta_{T} \stackrel{\sim}{\longrightarrow} \oplus_{v \in T} \mathbb{Z}[G] /\left(1-q^{d_{v}} \cdot \sigma_{v}^{-1}\right) .
$$


Since $\delta_{T}(0)=\prod_{v \in T}\left(1-q^{d_{v}} \cdot \sigma_{v}^{-1}\right)$, this implies right away that we have an equality of ideals in $\mathbb{Z}[G]$

$$
\operatorname{Fit}_{\mathbb{Z}[G]}\left(\Delta_{T}\right)=\mathbb{Z}[G] \cdot \delta_{T}(0) .
$$

According to $[\mathrm{P} 2], \S 3.1$, we have an exact sequence of $\mathbb{Z}[G]$-modules

$$
0 \rightarrow \Delta_{T} / \mu_{K} \rightarrow \operatorname{Pic}^{0}\left(X_{K}\right)_{T} \rightarrow \operatorname{Pic}^{0}\left(X_{K}\right) \rightarrow 0,
$$

where $\mu_{K}$ is embedded in $\Delta_{T}$ diagonally, i.e. $\zeta \longrightarrow(\zeta \bmod w)_{w \in T_{K}}$, for all $\zeta \in \mu_{K}$. Let $\chi \in \widehat{\Delta}\left(\mathbb{Q}_{\ell}\right) \backslash \widehat{\Delta / H}\left(\mathbb{Q}_{\ell}\right)$. Since $H$ acts trivially on $\mu_{K}$, we have $\left(\mu_{K} \otimes \mathbb{Z}_{\ell}\right)^{\chi}=\{1\}$. Therefore, we get the following exact sequence of $O_{\chi}[L]$-modules

$$
0 \rightarrow\left(\Delta_{T} \otimes \mathbb{Z}_{\ell}\right)^{\chi} \rightarrow\left(\operatorname{Pic}^{0}\left(X_{K}\right)_{T} \otimes \mathbb{Z}_{\ell}\right)^{\chi} \rightarrow\left(\operatorname{Pic}^{0}\left(X_{K}\right) \otimes \mathbb{Z}_{\ell}\right)^{\chi} \rightarrow 0 .
$$

Relations (7) and (6), combined with the exact sequence above, imply

$$
\delta_{T}(0)^{\chi} \cdot \operatorname{det}_{O_{\chi}[L]}\left(1-F_{*} \mid \mathrm{H}_{1}\left(\overline{X_{K}}, \mathbb{Z}_{\ell}\right)^{\chi}\right) \in \operatorname{Fit}_{O_{\chi}[L]}\left(\left(\operatorname{Pic}^{0}\left(X_{K}\right)_{T} \otimes \mathbb{Z}_{\ell}\right)^{\chi}\right)
$$

(see $\S 1.3)$. On the other hand, according to [P2], §3.1, we have an exact sequence of $\mathbb{Z}[G]$-modules

$$
\operatorname{Pic}^{0}\left(X_{K}\right)_{T} \rightarrow A_{S_{0}, T} \stackrel{\text { deg }}{\longrightarrow} \mathbb{Z} / d_{S_{0}} \mathbb{Z} \rightarrow 0,
$$

where "deg" denotes the usual $\mathbb{F}_{q}$-degree map at the level of divisors in $K, d_{S_{0}}$ is the greatest common divisor of $\left\{d_{v} \mid v \in S_{0}\right\}$, and $G$ acts trivially on $\mathbb{Z} / d_{S_{0}} \mathbb{Z}$. Let $\chi \in \widehat{\Delta}\left(\mathbb{Q}_{\ell}\right) \backslash \widehat{\Delta / H}\left(\mathbb{Q}_{\ell}\right)$. Since $H$ acts trivially on $\mathbb{Z} / d_{S_{0}} \mathbb{Z}$ and $\left.\chi\right|_{H} \neq \mathbf{1}_{H}$, we have $\left(\left(\mathbb{Z} / d_{S_{0}} \mathbb{Z}\right) \otimes \mathbb{Z}_{\ell}\right)^{\chi}=\{0\}$. Therefore, the exact sequence above leads to an exact sequence of $O_{\chi}[L]$-modules

$$
\left(\operatorname{Pic}^{0}\left(X_{K}\right)_{T} \otimes \mathbb{Z}_{\ell}\right)^{\chi} \rightarrow\left(A_{S_{0}, T} \otimes \mathbb{Z}_{\ell}\right)^{\chi} \rightarrow 0 .
$$

If we combine (8) above with the last exact sequence, we obtain

$$
\delta_{T}(0)^{\chi} \cdot \operatorname{det}_{O_{\chi}[L]}\left(1-F_{*} \mid \mathrm{H}_{1}\left(\overline{X_{K}}, \mathbb{Z}_{\ell}\right)^{\chi}\right) \in \operatorname{Fit}_{O_{\chi}[L]}\left(\left(A_{S_{0}, T} \otimes \mathbb{Z}_{\ell}\right)^{\chi}\right) .
$$

The last relation combined with the obvious equality Fit $_{O_{\chi}[L]}\left(\left(A_{S_{0}, T} \otimes \mathbb{Z}_{\ell}\right)^{\chi}\right)=$ $\left(\mathrm{Fit}_{\mathbb{Z}_{\ell}[G]}\left(A_{S_{0}, T} \otimes \mathbb{Z}_{\ell}\right)\right)^{\chi}$ concludes the proof of Proposition 3.4.

Proposition 3.4 combined with equalities (2) shows that relations (1) hold true for all $\chi \in \widehat{\Delta}\left(\mathbb{Q}_{\ell}\right) \backslash \widehat{\Delta / H}\left(\mathbb{Q}_{\ell}\right)$, concluding the proof of Theorem 3.1 in Case II as well.

Remark. Although not necessary for our current considerations, it is worth noting that, in the present context, the isomorphisms (3) lead to exact sequences of $\mathbb{Z}_{\ell}[G]$ modules

$$
\begin{aligned}
0 & \rightarrow \mathrm{H}_{2}\left(\overline{X_{K}}, \mathbb{Z}_{\ell}\right) \stackrel{1-F_{*}}{\longrightarrow} \mathrm{H}_{2}\left(\overline{X_{K}}, \mathbb{Z}_{\ell}\right) \rightarrow \mu_{K} \otimes \mathbb{Z}_{\ell} \rightarrow 0 \\
0 & \rightarrow \mathbb{Z}_{\ell} \rightarrow \mathrm{H}_{0}\left(\overline{X_{K}}, \mathbb{Z}_{\ell}\right) \stackrel{1-F_{*}}{\longrightarrow} \mathrm{H}_{0}\left(\overline{X_{K}}, \mathbb{Z}_{\ell}\right) \rightarrow \mathbb{Z}_{\ell} \rightarrow 0,
\end{aligned}
$$

where $G$ acts trivially on the two terms equal to $\mathbb{Z}_{\ell}$ in the second sequence above. These are the analogues of the exact sequence (5) involving the 1st $\ell$-adic homology group of $\overline{X_{K}}$, at the levels of the 2 nd and 0th $\ell$-adic homology groups of $\overline{X_{K}}$, respectively. 


\section{The $p$-primary component of the Rubin-Stark Conjecture.}

As usual, let $(K / k, S, T, r)$ be a set of data satisfying hypotheses $\left(H_{r}\right)$, where $k$ is a characteristic $p$ function field. As usual, let $V=\left(v_{1}, v_{2}, \ldots, v_{r}\right)$ be an ordered $r$-tuple of distinct primes in $S$ which split completely in $K / k, W=$ $\left(w_{1}, w_{2}, \ldots, w_{r}\right)$ with $w_{i} \mid v_{i}$ in $K$, for all $i$, and $S_{0}=S \backslash\left\{v_{1}, v_{2}, \ldots, v_{r}\right\}$. In [P5] (see also [P6]) we show that the $p$-part of the Strong Brumer-Stark Conjecture $\mathbb{Z}_{(p)} S B r S t\left(K / k, S_{0}, T\right)$ and the $p$-part of the Strong Rubin-Stark Conjecture $\mathbb{Z}_{(p)} S B(K / k, S, T, r)$ are false, in general.

However, the main result in $[\mathrm{T}]$ implies that the $p$-part of the Rubin-Stark Conjecture $\mathbb{Z}_{(p)} B(K / k, S, T, r)$ holds true. Since [T] lacks a detailed proof of this implication, we use this section to rewrite the main result in $[\mathrm{T}]$ by using the notations and terminology specific to the Rubin-Stark Conjecture (see Theorem 4.1 below) and present a detailed proof of $\mathbb{Z}_{(p)} B(K / k, S, T, r)$, as a consequence (see Theorem 4.3 below).

Let $L / K$ be a profinite, abelian extension of $K$, such that $L / k$ is abelian, unramified outside $S$, and $H:=G(L / K)$ has no torsion. Let $\Gamma:=G(L / k)$. We denote by $\mathbb{Z}[[\Gamma]]$ and $\mathbb{Z}[[H]]$ the rings of integral measures on $\Gamma$ and $H$, respectively. For each finite extension $M / k$, with $M \subseteq L$, we have a Stickelberger element $\Theta_{M / k, S, T}(0) \in \mathbb{Z}[G(M / k)]$, associated to $M / k, S$ and $T$ as in $\S 1.2$. Since these Stickelberger elements are coherent with respect to the maps in the projective limit above, they give rise to an element $\Theta_{L / k, S, T}(0):=\varliminf_{M / k, S, T}(0)$ in $\mathbb{Z}[[\Gamma]]$.

For every finite extension $M / k$, with $M \subseteq L$, let $I(G(M / K))$ denote the usual augmentation ideal of the group ring $\mathbb{Z}[G(M / K)]$ and let $I_{G(M / k)}$ be the kernel of the canonical projection $\mathbb{Z}[G(M / k)] \rightarrow \mathbb{Z}[G]$. We let $I(H):=\varliminf I(G(M / K))$ and $I_{H}:=\varliminf_{G(M / k)}$. Obviously, $I_{H}$ is the kernel of the natural projection $\mathbb{Z}[[\Gamma]] \rightarrow$ $\mathbb{Z}[G]$ and is generated as a $\mathbb{Z}[[\Gamma]]$-module by $I(H)$. For every positive integer $n$, the quotient $I_{H}^{n} / I_{H}^{n+1}$ has a natural $\mathbb{Z}[G]$-module structure given by

$$
g \cdot \widehat{j}:=\widehat{\widetilde{g} \cdot j}
$$

for all $g \in G$ and $j \in I_{H}^{n}$, where "ح" denotes classes modulo $I_{H}^{n+1}$ and $\widetilde{g}$ is an arbitrary element of $\Gamma$ which is mapped to $g$ via the canonical surjective morphism $\Gamma \rightarrow G$. One has natural isomorphisms of $\mathbb{Z}[G]$-modules

$$
\xi_{n}: I(H)^{n} / I(H)^{n+1} \otimes \mathbb{Z}[G] \stackrel{\sim}{\longrightarrow} I_{H}^{n} / I_{H}^{n+1},
$$

given by $\xi_{n}(\widehat{\iota} \otimes g)=\widehat{\widetilde{g} \cdot \iota}$, for all $\iota \in I(H)^{n}$ and all $g \in G$.

For every $v \in V$ and $w \mid v$ in $K$, we denote by $K_{w}$ the completion of $K$ with respect to the valuation associated to $w$. We let $\lambda_{w}: U_{S, T} \longrightarrow I(H) / I(H)^{2}$ be the composition:

$$
\lambda_{w}: U_{S, T} \longrightarrow K_{w}^{\times} \stackrel{\ell_{w}}{\longrightarrow} H \stackrel{\sim}{\longrightarrow} I(H) / I(H)^{2},
$$

where the left-most map is the usual inclusion, $\ell_{w}$ is the local Artin reciprocity map, and the right-most map is the group isomorphism which sends $h$ to the class of $1-h$ in $I(H) / I(H)^{2}$, for all $h \in H$. Now, one can define a $\mathbb{Q}[G]$-linear regulator map:

$$
R_{W, L}: \mathbb{Q}^{r} \wedge_{\mathbb{Z}[G]} U_{S, T} \longrightarrow I(H)^{r} / I(H)^{r+1} \otimes \mathbb{Q}[G] \stackrel{\xi_{r} \otimes \mathbf{1}_{\mathbb{Q}}}{\sim} \mathbb{Q} I_{H}^{r} / I_{H}^{r+1}
$$


by letting $R_{W, L}\left(u_{1} \wedge \ldots \wedge u_{r}\right)=\xi_{r}\left(\operatorname{det}\left(-\sum_{g \in G} \lambda_{w_{i}}\left(u_{j}^{g^{-1}}\right) \otimes g\right)\right)$, for all $u_{1}, \ldots, u_{r} \in$ $U_{S, T}$, and extending by $\mathbb{Q}$-linearity. The determinant above is viewed inside the graded algebra of $\mathbb{Z}[[\Gamma]]$ with respect to the ideal $I_{H}$, defined by

$$
\operatorname{gr}_{I_{H}} \mathbb{Z}[[\Gamma]]:=\bigoplus_{n \geq 0} I_{H}^{n} / I_{H}^{n+1}
$$

For every $\phi_{1}, \ldots, \phi_{r} \in \operatorname{Hom}_{\mathbb{Z}[G]}\left(U_{S, T}, I_{H} / I_{H}^{2}\right)$, one can define a $\mathbb{Q}[G]$-linear map

$$
\phi_{1} \wedge \ldots \wedge \phi_{r}: \mathbb{Q}^{r} \wedge_{\mathbb{Z}[G]} U_{S, T} \longrightarrow \mathbb{Q} I_{H}^{r} / I_{H}^{r+1},
$$

by letting $\phi_{1} \wedge \ldots \wedge \phi_{r}\left(u_{1} \wedge \ldots \wedge u_{r}\right)=\operatorname{det}\left(\phi_{i}\left(u_{j}\right)\right)$, for all $u_{1}, \ldots, u_{r} \in U_{S, T}$. Also, one can define a sub-lattice (i.e. a $\mathbb{Z}[G]$-submodule) of $\mathbb{Q}^{r} \wedge_{\mathbb{Z}[G]} U_{S, T}$ by setting

$$
\Lambda_{S, T, L}:=\left\{\varepsilon \in\left(\mathbb{Q}^{r}{ }_{\mathbb{Z}[G]} U_{S, T}\right)_{r, S} \mid \begin{array}{l}
\phi_{1} \wedge \ldots \wedge \phi_{r}(\varepsilon) \in I_{H}^{r} / I_{H}^{r+1} \\
\forall \phi_{1}, \ldots, \phi_{r} \in \operatorname{Hom}_{\mathbb{Z}[G]}\left(U_{S, T}, I_{H} / I_{H}^{2}\right)
\end{array}\right\} .
$$

The main result of $[\mathrm{T}]$ can be stated as follows.

Theorem 4.1 (Tan). Assume that $(K / k, S, T, r)$ satisfies hypotheses $\left(H_{r}\right)$. Then, for all pro-p extensions $L / K$ as above, the following hold true.

(1) $\Theta_{L / k, S, T}(0) \in I_{H}^{r}$.

(2) There exists a unique $\varepsilon_{S, T, L} \in \Lambda_{S, T, L}$ such that

$$
\Theta_{L / k, S, T}(0) \bmod I_{H}^{r+1}=R_{W, L}\left(\varepsilon_{S, T, L}\right) .
$$

Lemma 4.2. Let $L / K$ be an extension as above. If the Galois group $H$ of $L / K$ is isomorphic to the additive group $\mathbb{Z}_{p}^{+}$of p-adic integers, then one has an equality

$$
\Lambda_{S, T, L}=\mathbb{Z}_{(p)} \Lambda_{S, T},
$$

where $\Lambda_{S, T}$ is Rubin's lattice corresponding to the set of data $(K / k, S, T, r)$.

Proof. For any finite, cyclic group $M$ of generator $\sigma$, and any $n \in \mathbb{Z}_{\geq 1}$, one has a group-isomorphism depending on the chosen generator $\sigma$,

$$
\rho_{(M), \sigma}^{(n)}: I(M)^{n} / I(M)^{n+1} \stackrel{\sim}{\longrightarrow} M,
$$

sending the class $m \cdot \widehat{(1-\sigma})^{n} \in I(M)^{n} / I(M)^{n+1}$ of $m \cdot(1-\sigma)^{n} \in I(M)^{n}$ to $\sigma^{m} \in M$, for all $m \in \mathbb{Z}$. Since $H \stackrel{\sim}{\longrightarrow} \mathbb{Z}_{p}$ is pro-cyclic (i.e. a projective limit of finite cyclic groups), if one fixes a topological generator $\sigma$ of $H$ and takes projective limits, one obtains similar (continuous) isomorphisms

$$
\rho_{(H), \sigma}^{(n)}: I(H)^{n} / I(H)^{n+1} \stackrel{\sim}{\longrightarrow} H \stackrel{\sim}{\longrightarrow} \mathbb{Z}_{p},
$$

for all $n \in \mathbb{Z}_{\geq 1}$, uniquely determined by the equality

$$
\rho_{(H), \sigma}^{(n)}\left(m \cdot \widehat{(1-\sigma)^{n}}\right)=m,
$$


for all $n, m \in \mathbb{Z}$. Therefore, we have isomorphisms of $\mathbb{Z}_{p}[G]$-modules

$$
\begin{aligned}
\rho_{H, \sigma}^{(n)}: I_{H}^{n} / I_{H}^{n+1} & \stackrel{\xi_{n}^{-1}}{\longrightarrow} I(H)^{n} / I(H)^{n+1} \otimes \mathbb{Z}[G] \\
& \stackrel{\rho_{H, \sigma}^{(n)} \otimes \mathbf{1}}{\longrightarrow} \mathbb{Z}_{p} \otimes \mathbb{Z}[G] \stackrel{\sim}{\longrightarrow} \mathbb{Z}_{p}[G],
\end{aligned}
$$

for all $n \in \mathbb{Z}_{\geq 1}$. If we set $n=1$, we obtain the following group-isomorphisms

$$
\begin{aligned}
\operatorname{Hom}_{\mathbb{Z}[G]}\left(U_{S, T}, I_{H} / I_{H}^{2}\right) \stackrel{\sim}{\longrightarrow} \operatorname{Hom}_{\mathbb{Z}[G]}\left(U_{S, T}, \mathbb{Z}_{p}[G]\right) \\
\stackrel{\sim}{\longrightarrow} \operatorname{Hom}_{\mathbb{Z}[G]}\left(U_{S, T}, \mathbb{Z}[G]\right) \otimes \mathbb{Z}_{p} .
\end{aligned}
$$

Now, the lattice equality $\Lambda_{S, T, L}=\Lambda_{S, T} \otimes \mathbb{Z}_{(p)}$ follows immediately from the definitions of the two lattices, isomorphisms (10) and the elementary observation that $\mathbb{Q}[G] \cap \mathbb{Z}_{p}[G]=\mathbb{Z}_{(p)}[G]$.

As a consequence of Tan's Theorem 4.1 and Lemma 4.2 above, we prove the following (see $[\mathrm{T}]$ as well).

Theorem 4.3. If the set of data $(K / k, S, T, r)$ satisfies hypotheses $\left(H_{r}\right)$, then the p-primary component of the Rubin-Stark Conjecture $\mathbb{Z}_{(p)} B(K / k, S, T, r)$ holds true.

Proof. For a given set of data $(K / k, S, T, r)$ satisfying hypotheses $\left(H_{r}\right)$, a particular extension $L / K$ which satisfies the hypotheses of Lemma 4.2 is $L^{(p)} / K$, where $L^{(p)}$ is the maximal pro- $p$ constant field extension of $K$, i.e. the field compositum of $K$ with $\mathbb{F}_{q^{p^{\alpha}}}$, for all $\alpha \in \mathbb{Z}_{\geq 1}$. The Galois group $H^{(p)}:=G\left(L^{(p)} / K\right)$ is isomorphic to $\mathbb{Z}_{p}$ and has a distinguished topological generator $\sigma_{q^{n}}$, satisfying $\sigma_{q^{n}}(\zeta)=\zeta^{q^{n}}$, for all $\zeta \in \mu_{L^{(p)}}$, where $\mathbb{F}_{q}$ and $\mathbb{F}_{q^{n}}$ are the exact field of constants of $k$ and $K$, respectively. In order to simplify notations, we will denote $L^{(p)}$ by $L, H^{(p)}$ by $H$, and $\sigma_{q^{n}}$ by $\sigma$ throughout the proof of Theorem 4.3. The extension $L^{(p)} / K$ is unramified everywhere and, for a prime $w$ of $K$ of degree $d_{w}$ over $\mathbb{F}_{q^{n}}$, the Frobenius morphism $\sigma_{w}(L / K)$ associated to $w$ in $L / K$ is given by

$$
\sigma_{w}:=\sigma_{w}(L / K)=\sigma^{d_{w}} .
$$

Consequently, the local Artin reciprocity map $\ell_{w}: K_{w}^{\times} \longrightarrow H$ is given by

$$
\ell_{w}(x)=\left(\sigma_{w}^{-1}\right)^{\operatorname{ord}_{w}(x)}=\sigma^{-d_{w} \cdot \operatorname{ord}_{w}(x)}, \text { for all } x \in K_{w}^{\times} .
$$

Therefore, the maps $\lambda_{w}$ are given by

$$
\lambda_{w}(x)=\left(\widehat{1-\sigma_{w}^{-1}}\right)=\left(1-\widehat{\sigma^{-d_{w} \cdot \operatorname{ord}_{w}}(x)}\right) \in I(H) / I(H)^{2}, \text { for all } x \in U_{S, T} .
$$

This leads to the following equality in $\mathbb{Q}_{p}[G]$

$$
\rho_{H, \sigma}^{(n)}\left(R_{W, L}\left(u_{1} \wedge \ldots \wedge u_{r}\right)\right)=\left(\prod_{i=1}^{r} d_{w_{i}}\right) \cdot \operatorname{det}\left(\sum_{g \in G} \operatorname{ord}_{w_{i}}\left(u_{j}^{g^{-1}}\right) \cdot g\right)
$$


for all $u_{1}, \ldots, u_{r} \in U_{S, T}$. Consequently, since they are $\mathbb{Q}[G]$-linear maps, Rubin's regulator $R_{W}$ and the regulator $R_{W, L}$ are related as follows.

$$
\rho_{H, \sigma}^{(r)}\left(R_{W, L}(\varepsilon)\right)=\frac{1}{\left(\log q^{n}\right)^{r}} \cdot R_{W}(\varepsilon), \text { for all } \varepsilon \in \mathbb{Q}^{\stackrel{r}{ }} \mathbb{Z}[G] U_{S, T} .
$$

On the other hand, since the extension $L / k$ is unramified and $K / k$ is totally split at $v_{i}$, for all $i=1, \ldots, r$, we have the following equality.

$$
\Theta_{L / k, S, T}(0)=\prod_{i=1}^{r}\left(1-\sigma_{w_{i}}^{-1}\right) \cdot \Theta_{L / k, S_{0}, T}(0)
$$

Therefore, we have the following equality in $\mathbb{Z}_{p}[G]$,

$$
\rho_{H, \sigma}^{(r)}\left(\Theta_{L / k, S, T}(0) \quad \bmod I_{H}^{r+1}\right)=\left(\prod_{i=1}^{r} d_{w_{i}}\right) \cdot \Theta_{S_{0}, T}(0)=\frac{1}{\left(\log q^{n}\right)^{r}} \cdot \Theta_{S, T}^{(r)}(0)
$$

Tan's Theorem 4.1 applied to $L / K$, combined with the equalities (12) and (11) and Lemma 4.2 above, leads to the conclusion that there exists a unique element $\varepsilon_{L, S, T} \in \mathbb{Z}_{(p)} \Lambda_{S, T}$, such that

$$
R_{W}\left(\varepsilon_{L, S, T}\right)=\Theta_{S, T}^{(r)}(0) .
$$

Consequently, conjecture $\mathbb{Z}_{(p)} B(K / k, S, T, r)$ holds true.

\section{The Rubin-Stark Conjecture for subextensions of $k_{p \infty} / k$}

In what follows, $k$ is a fixed, arbitrary function field of characteristic $p$. We fix an abelian closure $k^{\mathrm{ab}}$ of $k$. We denote by $k_{p}$ and $k_{\infty}$ the maximal pro- $p$ abelian extension of $k$ and the maximal constant field extension of $k$ inside $k^{\text {ab }}$, respectively. By definition, $k_{p \infty}$ is the compositum $k_{p} \cdot k_{\infty}$ of $k_{p}$ and $k_{\infty}$ inside $k^{\text {ab }}$. Class-field theory combined with the fact that Leopoldt's conjecture is true in characteristic $p$ (see $[\mathrm{Ki}]$ ) implies that, as a topological group, $G\left(k_{p} / k\right)$ is isomorphic to $\mathbb{Z}_{p}^{\aleph_{0}}$ (the direct product of countably many copies of $\mathbb{Z}_{p}^{+}$). Also, the very definition of $k_{\infty}:=k\left(\mu_{k^{\mathrm{ab}}}\right)$ implies that, as a topological group, $G\left(k_{\infty} / k\right)$ is isomorphic to the profinite completion $\widehat{\mathbb{Z}}$ of $\mathbb{Z}$, given by

$$
\widehat{\mathbb{Z}}=\varliminf_{n \geq 1}(\mathbb{Z} / n \mathbb{Z}) .
$$

Obviously, we have a topological group isomorphism $G\left(k_{p} \cap k_{\infty} / k\right) \stackrel{\sim}{\longrightarrow} \mathbb{Z}_{p}$. Consequently, we have a topological group isomorphism

$$
G\left(k_{p \infty} / k\right) \stackrel{\sim}{\longrightarrow} \mathbb{Z}_{p}^{\aleph_{0}} \times \prod_{\ell \neq p} \mathbb{Z}_{\ell},
$$

where the product is taken with respect to all the primes $\ell \neq p$. 
Lemma 5.1. Let $K / k$ be a finite extension, with $K \subseteq k_{p \infty}$. Then $K / k$ is an $\ell$-constant extension, for all primes $\ell \neq p$.

Proof. Let $H:=G\left(K / k\left(\mu_{K}\right)\right)$. Since, by definition, $k_{\infty}=k\left(\mu_{k^{\mathrm{ab}}}\right)$, we have $k\left(\mu_{K}\right)=K \cap k_{\infty}$. Consequently, via the restriction map at the level of Galois groups, $H$ can be viewed as a finite quotient of $G\left(k_{p \infty} / k_{\infty}\right) \stackrel{\sim}{\longrightarrow} \mathbb{Z}_{p}^{\aleph_{0}}$. Consequently, $H$ is a $p$-group. Therefore $\ell \nmid|H|$, for all primes $\ell \neq p$. Proposition 2.3.1(2) implies that $K / k$ is $\ell$-constant, for all primes $\ell \neq p$.

Theorem 5.2. Let $(K / k, S, T, r)$ be a set of data satisfying hypotheses $\left(H_{r}\right)$, such that $K \subseteq k_{p \infty}$. Let $v_{1}, v_{2}, \ldots, v_{r}$ be $r$ distinct primes in $S$ which split completely in $K / k$, and let $S_{0}:=S \backslash\left\{v_{1}, \ldots, v_{r}\right\}$. Then,

(1) The Rubin-Stark Conjecture $B(K / k, S, T, r)$ is true.

(2) At primes $\ell \neq p$, the $\ell$-primary components of the strong forms of the Rubin-Stark Conjecture $\mathbb{Z}_{(\ell)} S B(K / k, S, T, r)$ and the Brumer-Stark Conjecture $\mathbb{Z}_{(\ell)} \operatorname{SBr} S t\left(K / k, S_{0}, T\right)$ hold true.

(3) The p-primary components of the strong forms of the Rubin-Stark Conjecture $\mathbb{Z}_{(p)} S B(K / k, S, T, r)$ and the Brumer-Stark Conjecture $\mathbb{Z}_{(p)} \operatorname{SBr} S t\left(K / k, S_{0}, T\right)$ are false, in general.

Proof. (1) This is a direct consequence of Lemma 5.1, Theorem 4.2, Theorem 4.3, and Remark 2 in $\S 1.5$.

(2) This is a direct consequence of Lemma 5.1, and Theorems 3.1 and 3.2.

(3) In [P5] (see also [P6]), we construct an example of a set of data $(K / k, S, T, 2)$ satisfying hypotheses $\left(H_{2}\right)$, with $G(K / k) \stackrel{\sim}{\longrightarrow}(\mathbb{Z} / p \mathbb{Z})^{3}$ (therefore $\left.K \subseteq k_{p} \subseteq k_{p \infty}\right)$, for which $\mathbb{Z}_{(p)} S B(K / k, S, T, 2)$ and $\mathbb{Z}_{(p)} S B r S t\left(K / k, S_{0}, T\right)$ are false.

Proposition 5.3 below shows that in fact Theorem 5.2 settles the Rubin-Stark Conjecture for a very large class of abelian extensions $K / k$ of a given function field $k$.

Proposition 5.3. Let $k$ be a characteristic $p$ function field, and $k^{\mathrm{ab}}$ and $k_{p \infty}$ as above. Then the extension $k^{\mathrm{ab}} / k_{p \infty}$ is quasi-finite, in the following sense: if $S$ is an arbitrary, finite (possibly empty) set of primes in $k$ and $k_{S}^{\mathrm{ab}}$ is the maximal abelian extension of $k$ unramified outside $S$, then the index $\left[k_{S}^{\mathrm{ab}}: k_{S}^{\mathrm{ab}} \cap k_{p \infty}\right]$ is finite.

Proof (sketch). In what follows, $J_{k}$ denotes the idèle group of $k$, and $J_{k}^{(0)}$ the subgroup of $J_{k}$ consisting of the idèles of degree 0 . If $v$ is a prime in $k$, we denote by $k_{v}$ the completion of $k$ at $v$, by $U_{v}$ and $U_{v}^{(1)}$ the groups of units and principal units in $k_{v}^{\times}$, respectively. The group $U_{v}^{(1)}$ is a pro- $p$ group, topologically isomorphic to $\mathbb{Z}_{p}^{\aleph_{0}}$. We have an isomorphism of topological groups

$$
U_{v} \stackrel{\sim}{\longrightarrow} U_{v}^{(1)} \times \mathbb{F}_{q}(v)^{\times} .
$$

Let us fix a finite set $S$ of primes in $k$. Since $k_{\infty} / k$ is unramified everywhere, we have $k_{\infty} \subseteq k_{S}^{\mathrm{ab}}$. Therefore, we have an equality of Galois groups

$$
G\left(k_{S}^{\mathrm{ab}} / k_{S}^{\mathrm{ab}} \cap k_{p \infty}\right)=\operatorname{ker}\left(G\left(k_{S}^{\mathrm{ab}} / k_{\infty}\right) \stackrel{\mathrm{res}}{\rightarrow} G\left(k_{S}^{\mathrm{ab}} \cap k_{p \infty} / k_{\infty}\right)\right),
$$

where "res" denotes the usual restriction map. On the other hand, the global Artin reciprocity map establishes group isomorphisms between $J_{k}^{0} / k^{\times} \cdot \prod_{v \notin S} U_{v}$ 
and $G\left(k_{S}^{\mathrm{ab}} / k_{\infty}\right)$ and the maximal pro- $p$ quotient $\left(J_{k}^{0} / k^{\times} \cdot \prod_{v \notin S} U_{v}\right)^{(p)}$ of $J_{k}^{0} / k^{\times}$. $\prod_{v \notin S} U_{v}$ and $G\left(\left(k_{S}^{\mathrm{ab}} \cap k_{p \infty}\right) / k_{\infty}\right)$, respectively. In what follows, if $\mathcal{G}$ is a profinite group, we denote by $\mathcal{G}^{(p)}$ its maximal pro- $p$ quotient. Since the usual divisor map induces a group isomorphism $J_{k}^{0} / k^{\times} \cdot \prod_{v} U_{v} \stackrel{\sim}{\longrightarrow} \operatorname{Pic}^{0}(k)$, we have a commutative diagram of short exact sequences of topological groups.

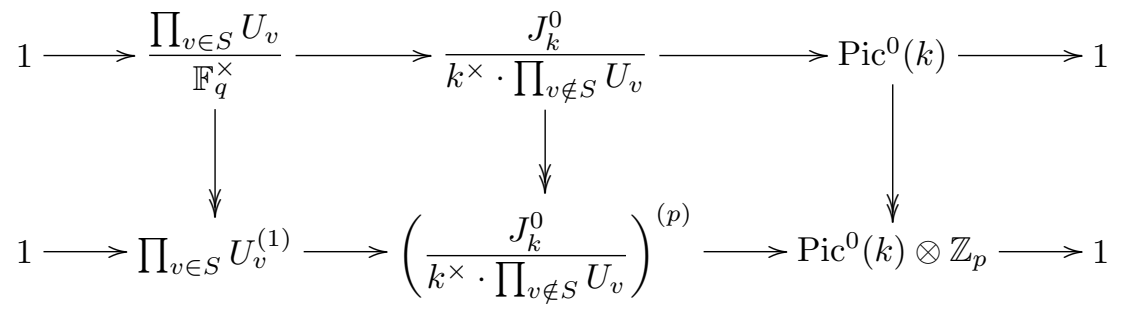

The lower exact sequence in the diagram above is just the exact sequence of the maximal pro- $p$ quotients of the terms in the upper exact sequence. If we apply the snake lemma to the above commutative diagram, we obtain the following exact sequence of abelian groups, linking the kernels of the vertical maps in the commutative diagram above.

$$
1 \longrightarrow \frac{\prod_{v \in S} \mathbb{F}_{q}(v)^{\times}}{\mathbb{F}_{q}^{\times}} \longrightarrow G\left(k_{S}^{\mathrm{ab}} / k_{S}^{\mathrm{ab}} \cap k_{p \infty}\right) \longrightarrow \frac{\operatorname{Pic}^{0}(k)}{\operatorname{Pic}^{0}(k) \otimes \mathbb{Z}_{p}} \longrightarrow 1
$$

Since the non-trivial end-terms in the above exact sequence are finite, the term in the middle is also finite. This concludes the proof of Proposition 5.3.

\section{A weaker form of the Rubin-Stark Conjecture}

Assume that the set of data $(K / k, S, r)$ satisfies conditions $\left(H_{r}\right) 1-3$. In [P4] (see also [P6]), we formulated a conjecture $C(K / k, S, r)$ which can also be viewed as an integral refinement of Stark's Conjecture for abelian $L$-functions of arbitrary order of vanishing at $s=0$. The reader can consult $[\mathrm{P} 4]$ and $[\mathrm{P} 6]$ for the exact statement. Conjecture $C(K / k, S, r)$ is (at least theoretically) slightly weaker than the Rubin-Stark Conjecture. The precise link between the two statements is given by the following theorem, proved in [P4] (see Theorem 5.5.1.)

Theorem 6.1. Let us assume that the set of data $(K / k, S, r)$ satisfies hypotheses $\left(H_{r}\right) 1-3$. Let $\mathcal{T}$ be the set consisting of all those sets $T$, such that $(K / k, S, T, r)$ satisfies hypotheses $\left(H_{r}\right)$. Then, the following hold true, for all prime numbers $\ell$.

$$
\left\{\begin{array}{c}
\mathbb{Z}_{(\ell)} B(K / k, S, T, r), \\
\quad \text { for all } T \in \mathcal{T}
\end{array}\right\} \Longrightarrow Z_{(\ell)} C(K / k, S, r) .
$$

(2) If $K / k$ is $\ell$-admissible, then

$$
\left\{\begin{array}{c}
\mathbb{Z}_{(\ell)} B(K / k, S, T, r), \\
\text { for all } T \in \mathcal{T}
\end{array}\right\} \Longleftrightarrow Z_{(\ell)} C(K / k, S, r) .
$$

Two immediate consequences are the following. 
Corollary 6.2. Assume that $K / k$ is an arbitrary abelian extension of characteristic $p$ function field, and the set of data $(K / k, S, r)$ satisfies hypotheses $\left(H_{r}\right) 1-3$. Then, the following hold true.

(1)

$$
\left\{\begin{array}{c}
\mathbb{Z}_{(p)} B(K / k, S, T, r) \\
\quad \text { for all } T \in \mathcal{T}
\end{array}\right\} \Longleftrightarrow \mathbb{Z}_{(p)} C(K / k, S, r)
$$

(2) Conjecture $\mathbb{Z}_{(p)} C(K / k, S, r)$ holds true.

Proof. (1) Since $\mu_{K}^{(p)}=1, K / k$ is $p$-admissible. Therefore, Theorem $6.1(2)$ for $\ell:=p$ implies $(1)$.

(2) This is a direct consequence of (1) and Theorem 4.3 above.

Corollary 6.3. Let $K / k$ be an abelian extension of characteristic $p$ function fields, such that $K \subseteq k_{p \infty}$. Assume that the set of data $(K / k, S, r)$ satisfies hypotheses $\left(H_{r}\right) 1-3$. Then

$$
\left\{\begin{array}{c}
B(K / k, S, T, r) \\
\text { for all } T \in \mathcal{T}
\end{array}\right\} \Longleftrightarrow C(K / k, S, r) .
$$

(2) Conjecture $C(K / k, S, r)$ holds true.

Proof. (1) According to Lemma 5.1 and Lemma 2.3.3(1), the hypothesis $K \subseteq k_{p \infty}$ implies that $K / k$ is $\ell$-admissible, for all primes $\ell \neq p$. However, as remarked in the proof of Corollary $6.2, K / k$ is automatically $p$-admissible. In light of these observations, (1) is a direct consequence of Theorem 6.1(2).

(2) This is a direct consequence of (1) and Theorem 5.2(1) above.

\section{REFERENCES}

[Bu1] Burns, D., Equivariant Tamagawa numbers and refined abelian Stark conjectures, J. Math. Sci. Univ. Tokyo 10 (2003), no. 2, 225-259.

[Bu2] Burns, D., On the values of equivariant Zeta functions of curves over finite fields, preprint submitted for publication.

[Bu3] Burns, D., Congruences between derivatives of abelian L-functions at $s=0$ (preliminary version), preprint 2004.

[BuGr] Burns, D. and Greither, C., On the equivariant Tamagawa number conjecture for Tate motives, Invent. Math. 153 (2003), no. 2, 303-359.

[CF] Cassels, J.W.S., and Fröhlich, A, Editors, Algebraic Number Theory (1967), Academic Press, London and New York.

[Gro1] Gross, B.H., p-adic L-series at $s=0$, J.Fac.Sci.Univ. Tokyo 28 (1981), 979-994.

[Gro2] Gross, B.H., On the values of abelian L-functions at $s=0$, J.Fac.Sci.Univ. Tokyo 35 (1988), 177-197.

[Gro3] Gross, B.H., Letter to John Tate, November 1993.

[H] Hayes, D.R., Stickelberger elements in function fields, Comp. Math. 55 (1985), 209-239.

[Ki] Kisilevsky, H, Multiplicative independence in function fields, Journal of Number Theory 44 (1993), 352-355.

[MW] Mazur, B. and Wiles, A., Class fields of abelian extensions of $\mathbb{Q}$, Invent. Math. 76 (1984), 179-330.

[P1] Popescu, C.D., On a refined Stark conjecture for function fields (1996), Ph.D. Thesis, Ohio State University.

[P2] Popescu, C.D., On a refined Stark conjecture for function fields, Comp. Math. 116 (1999), 321-367.

[P3] Popescu, C.D., Gras-type conjectures for function fields, Comp. Math. 118 (1999), no. 3, 263-290. 
[P4] Popescu, C.D., Base change for Stark-type conjectures "over $\mathbb{Z}$ ", J. Reine Angew. Math 542 (2002), 85-111.

[P5] Popescu, C.D., Stark's Question and a strong form of Brumer's conjecture extrapolated to function fields, to appear in Compositio Math. (2004).

[P6] Popescu, C.D., Rubin's integral refinement of the abelian Stark Conjecture, in "The Stark Conjectures: Recent Work and New Directions", D. Burns, C. Popescu, J. Sands, D. Solomon, Editors, Contemporary Mathematics, 2004.

$[\mathrm{Ru}] \quad$ Rubin, K., A Stark conjecture "over $\mathbb{Z}$ " for abelian L-functions with multiple zeros, Annales de L'Institut Fourier 46 (1996), 33-62.

[St] Stark, H., L-functions at $s=1$, I, II, III, IV, Adv. in Math. 7 (1971), 301-343; 17 (1975), 60-92; 22 (1976), 64-84; 35 (1980), 197-235.

[Sw] Swan, R. G., Algebraic K-theory, Lecture Notes in Mathematics 76 (1968), Springer, Berlin.

[T] Tan, K.S., Generalized Stark formulae over function fields, preprint submitted for publication.

[Ta1] Tate, J., The cohomology groups of tori in finite Galois extensions of number fields, Nagoya Math. J. 27 (1966), 709-719.

[Ta2] Tate, J., Brumer-Stark-Stickelberger, Séminaire de Théorie des Nombres, Univ. de Bordeaux (1980-1981), exp. 24.

[Ta3] Tate, J., On Stark's conjecture on the behavior of $L(s, \chi)$ at $s=0$, J. Fac. Sci. Univ. Tokyo 28 (1982), 963-978.

[Ta4] Tate, J., Les conjectures de Stark sur les fonctiones L d'Artin en $s=0$, Progr. in Math. 47 (1984), Boston Birkhäuser.

University of California, San Diego, Department of Mathematics, 9500 Gilman Drive, La Jolla, CA 92093-0112, USA

E-mail address: cpopescu@math.ucsd.edu 